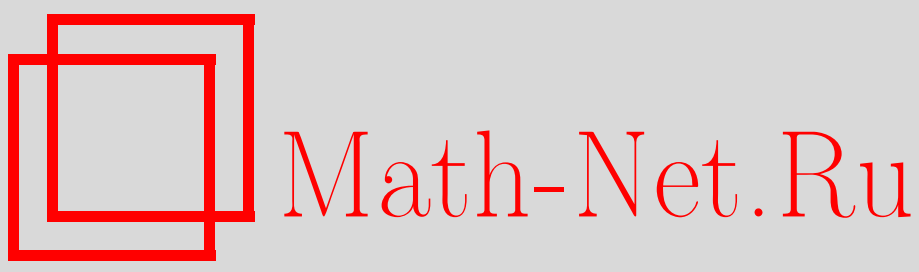

Ха Зуй Банг, Изучение свойств функций из пространства Орлича в зависимости от геометрии их спектра, Изв. РАН. Сер. матем., 1997, том 61, выпуск 2, 163-198

DOI: https://doi.org/10.4213/im120

Использование Общероссийского математического портала Math-Net.Ru подразумевает, что вы прочитали и согласны с пользовательским соглашением

http://www . mathnet.ru/rus/agreement

Параметры загрузки:

IP : 18.207 .199 .55

26 апреля 2023 г., 13:18:19 
УДК 517.9

\title{
Ха Зуй Банг \\ Изучение свойств функций из пространства Орлича в зависимости от геометрии их спектра
}

\begin{abstract}
Изучается геометрия спектра (носителя преобразования Фурье) функций из пространства Орлича $L_{\Phi}\left(\mathbb{R}^{n}\right)$ и доказано, в частности, что если $f \in L_{p}\left(\mathbb{R}^{n}\right)$, $1 \leqslant p<\infty$ и $f(x) \not \equiv 0$, то для любой точки спектра функции $f$ существует последовательность точек с ненулевыми компонентами из ее спектра, стремящихся к ней. Доказывается, что поведение последовательности норм Люксембурга производных функции полностью характеризуется ее спектром. Даются с помощью нового метода неравенства Никольского в норме Люксембурга для функций с произвольным спектром. Полученные результаты применяются для получения теорем типа Пэли-Винера-Шварца для необязательно выпуклых случаев и для изучения некоторых вопросов теории пространств Соболева-Орлича бесконечного порядка, развиваемой в последние годы Ю. А. Дубинским и его учениками.

Библиография: 31 наименование.
\end{abstract}

Целье функции экспоненциального типа, ограниченные на действительном пространстве $\mathbb{R}^{n}$, имеют свойства, аналогичные соответствующим свойствам тригонометрических полиномов. В то время как тригонометрические полиномы являются хорошим средством приближения периодических функций, целые функции экспоненциального типа могут служить средством приближения непериодических функций, заданных на $n$-мерном пространстве.

В данной работе мы изучим некоторые свойства целых функций экспоненциального типа (которые как функции от действительного переменного принадлежат пространству Орлича), обусловленные геометрией их спектра (носителя преобразования Фурье), и дадим некоторые их применения.

Пусть $f \in L_{p}\left(\mathbb{R}^{n}\right)$. Тогда ее преобразование Фурье $\hat{f}(\xi)$, вообще говоря, является обобщенной функцией (если $p>2$ ), поэтому совершенно неясна геометрия ее спектра. В $\S 1$ мы изучаем геометрию спектра функций пространства Орлича $L_{\Phi}\left(\mathbb{R}^{n}\right)$ и доказываем, в частности, что если $f \in L_{p}\left(\mathbb{R}^{n}\right), 1 \leqslant p<\infty$, и $f \not \equiv 0$, то для любой точки спектра функции $f$ сушествует последовательность точек с ненулевыми компонентами из ее спектра, стремяшаяся к ней. Это изучение имеет применения в последующих параграфах. В $\S 2$ мы изучаем поведение последовательности норм производных $\left\|D^{\alpha} f\right\|_{(\Phi)}, \alpha \geqslant 0$, и показываем, что оно полностью характеризуется спектром функции $f$. В $\S 3$ с помощью нового метода изучаем неравенства Никольского в норме пространства Орлича для функций с произвольным спектром. Следует отметить, что неравенства Никольского [1], [2], играющие очень важную роль в теории функций и имеющие обширные и важные приложения, были изучены в многочисленных работах (см., например, [1]-[5] и библиографию в [6]). В $\S 4$ мы применим результаты предыдуших параграфов для получения

Работа поддержана Национальной программой естественных наук Вьетнама.

$$
\text { (C) ХА ЗУЙ БАнГ } 1997
$$


теорем типа Пэли-Винера-Шварца для необязательно вьпуклых случаев и для изучения некоторых вопросов теории пространств Соболева-Орлича бесконечного порядка, развиваемой в последние годы Ю. А. Дубинским и его учениками.

Пользуясь случаем, автор выражает глубокую благодарность члену-корреспонденту РАН О.В. Бесову за указание на одну неточность в прежнем доказательстве теоремы 1 данной работы.

\section{§ 0. Необходимые сведения}

Пусть $\Phi(t):[0,+\infty) \rightarrow[0,+\infty]-$ произвольная функция Юнга, т.е. $\Phi(0)=0$, $\Phi(t) \geqslant 0, \Phi(t) \not \equiv 0$ и $\Phi(t)$ выпукла.

Функция

$$
\bar{\Phi}(t)=\sup _{s \geqslant 0}\{t s-\Phi(s)\}
$$

называется дополнительной функиией к функции Юнга $\Phi(t)$. Тогда $\bar{\Phi}(t)$ также является функцией Юнга. Из определения функции Юнга легко видеть, что $\Phi(t) / t$ не убывает на $[0,+\infty)$ и, подавно, $\Phi(t)$ не убывает.

Далее, пусть $G$ - некоторая область в $\mathbb{R}^{n}$ или тор $\mathbb{T}^{n}$. Через $L_{\Phi}(G)$ будем обозначать совокупность всех функций $u(x)$ таких, что ее норма Люксембурга

$$
\|u\|_{(\Phi)}=\inf \left\{\lambda>0: \int_{G} \Phi\left(\frac{|u(x)|}{\lambda}\right) d x \leqslant 1\right\}<\infty .
$$

Тогда $L_{\Phi}(G)$ является банаховым пространством и называется пространством Орлича. Норма Люксембурга эквивалентна норме Орлича $\|\cdot\|_{\Phi}$, причем

$$
\|u\|_{(\Phi)} \leqslant\|u\|_{\Phi} \leqslant 2\|u\|_{(\Phi)} .
$$

Напомним, что $\|\cdot\|_{(\Phi)}=\|\cdot\|_{p}$, если $1 \leqslant p<\infty$ и $\Phi(t)=t^{p}$, и $\|\cdot\|_{(\Phi)}=\|\cdot\|_{\infty}$, если $\Phi(t)=0$ при $0 \leqslant t \leqslant 1$ и $\Phi(t)=\infty$ при $t>1$ (см., например, [7]-[10]).

Лемма 1 [10]. Пусть $u \in L_{\Phi}\left(\mathbb{R}^{n}\right) u v \in L_{1}\left(\mathbb{R}^{n}\right)$. Тогда

$$
\|u * v\|_{\Phi} \leqslant\|u\|_{\Phi}\|v\|_{1}
$$

ЛЕмма 2 [10]. Пусть $u \in L_{\Phi}(G) u v \in L_{\bar{\Phi}}(G)$. Тогда

$$
\int_{G}|u(x) v(x)| d x \leqslant\|u\|_{\Phi}\|v\|_{\bar{\Phi}}
$$

Пусть $Q$ - некоторая область в $\mathbb{R}^{n}$ и $m \in \mathbb{Z}_{+}$. Обозначим через $W_{m, 2}(Q)$ пространство Соболева, т.е. пополнение $C^{m}(Q)$ по норме

$$
\|f\|_{m, 2}=\left(\sum_{|\alpha| \leqslant m}\left\|D^{\alpha} f\right\|_{L_{2}(Q)}^{2}\right)^{1 / 2},
$$

a $W_{m, 2}^{0}(Q)$ - пополнение $C_{0}^{\infty}(Q)$ по этой норме. 
Для $s \in \mathbb{R}$ положим

$$
H_{(s)}=\left\{f \in S^{\prime}:\|f\|_{(s)}=\left(\int_{\mathbb{R}^{n}}\left(1+|\xi|^{2}\right)^{s}|F f(\xi)|^{2} d \xi\right)^{1 / 2}<\infty\right\} .
$$

Тогда имеет место топологическое равенство $H_{(k)}=W_{k, 2}\left(\mathbb{R}^{n}\right)$, если $k \in \mathbb{Z}_{+}$(см., например, [11]-[13]).

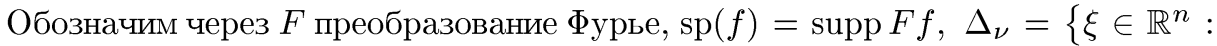
$\left.\left|\xi_{j}\right| \leqslant \nu_{j}, j=1, \ldots, n\right\}, D=\left(D_{1}, \ldots, D_{n}\right), D_{j}=-i \frac{\partial}{\partial x_{j}}, \nu_{j} \geqslant 0, j=1, \ldots, n$, $D^{\alpha}=D_{1}^{\alpha_{1}} \ldots D_{n}^{\alpha_{n}}$. Будем считать, что $\frac{0}{0}=1$ и $\frac{\lambda}{0}=\infty$ для $\lambda>0$.

\section{$\S 1$. Геометрия спектра}

Изучаем теперь геометрию спектра функций пространства Орлича $L_{\Phi}\left(\mathbb{R}^{n}\right)$.

TeOPEMA 1. Пусть $\Phi(t)>0$ для $t>0, f \in L_{\Phi}\left(\mathbb{R}^{n}\right), \quad f(x) \not \equiv 0 u \xi^{0} \in \mathbb{R}^{n}-$ произвольная точка. Тогда распределение Ff не может быть сосредоточенныц.м на гиперплоскостях $\xi_{j}=\xi_{j}^{0}, j=1, \ldots, n$.

ДоказАТЕЛЬСТво. Пусть $\nu \geqslant 0$ - такой вектор, что $\xi^{0} \in \Delta_{\nu}$, и функция $\widehat{\varphi}(\xi) \in$ $C_{0}^{\infty}\left(\mathbb{R}^{n}\right)$ такая, что $\widehat{\varphi}$ равна единице в некоторой окрестности $\Delta_{\nu}$. Тогда $F^{-1} \widehat{\varphi} \hat{f}=$ $\varphi * f \in L_{\Phi}\left(\mathbb{R}^{n}\right)$ в силу леммы 1 . Следовательно, нам достаточно доказать нашу теорему только для функций с ограниченным спектром.

Положим $\hat{h}(\xi)=\hat{f}\left(\xi-\xi^{0}\right)$. Тогда $h(x)=e^{i \xi^{0} x} f(x)$ принадлежит $L_{\Phi}\left(\mathbb{R}^{n}\right)$ и обладает ограниченным спектром.

Нам осталось доказать, что распределение $\hat{h}(\xi)$ не может быть сосредоточенным на гиперплоскостях $\xi_{j}=0, j=1, \ldots, n$. Докажем методом от противного. Пусть распределение $\hat{h}(\xi)$ сосредоточено на гиперплоскостях $\xi_{j}=0, j=1, \ldots, n$. Тогда положим для каждого $j=1, \ldots, n$

$$
G_{j}=\left\{\xi \in \mathbb{R}^{n}: \xi_{i} \neq 0, \quad i \in I \backslash\{j\}\right\},
$$

где $I=\{1, \ldots, n\}$. Тогда $G_{j}$ открыто. Для каждой функции $\psi(\xi)$ из $C_{0}^{\infty}\left(G_{j}\right)$ распределение $\psi(\xi) \hat{h}(\xi)$ сосредоточено на гиперплоскости $\xi_{j}=0$. Следовательно, учитьвая одно замечание о теореме 2.3.5, упомянутое в примере 5.1.2 из [12], получим

$$
F^{-1}(\psi \hat{h})(x)=\sum_{l=0}^{N} g_{l}\left(x_{1}, \ldots, x_{j-1}, x_{j+1}, \ldots, x_{n}\right)\left(-i x_{j}\right)^{l},
$$

где $N$-порядок распределения $\hat{h}(\xi)\left(N<\infty\right.$, так как $\operatorname{supp} \hat{h}$ компактно) и $\hat{g}_{l}\left(\xi_{1}, \ldots\right.$, $\left.\xi_{j-1}, \xi_{j+1}, \ldots, \xi_{n}\right), 0 \leqslant l \leqslant N,-$ распределения с компактным носителем.

Из леммы 3, формулируемой позже, имеем для некоторого $C>0$

$$
C^{-1}\left\|F^{-1}(\psi \hat{h})\right\|_{\infty} \leqslant\left\|F^{-1}(\psi \hat{h})\right\|_{(\Phi)}=\left\|F^{-1} \psi * h\right\|_{(\Phi)} \leqslant 2\left\|F^{-1} \psi\right\|_{1}\|h\|_{(\Phi)}<\infty .
$$

Следовательно, равенство (1) возможно, лишь когда $N=0$. Отсюда функция $F^{-1}(\psi \hat{h})(x)$ не зависит от $x_{j}$. 
Далее, $F^{-1}(\psi \hat{h})(x) \in L_{\Phi}\left(\mathbb{R}^{n}\right)$ в силу леммы 1. Следовательно, по определению

$$
\int_{\mathbb{R}^{n}}\left(\left|F^{-1}(\psi \hat{h})(x)\right| / \lambda\right) d x<\infty
$$

для некоторого $\lambda>0$. Отсюда получим

$$
\Phi\left(\left|F^{-1}(\psi \hat{h})(x)\right| / 2 \lambda\right) \equiv 0 .
$$

Действительно, пусть

$$
\Phi\left(\left|F^{-1}(\psi \hat{h})\left(x^{0}\right)\right| / 2 \lambda\right)>0
$$

для некоторой точки $x^{0}$. Из неубываемости $\Phi(t)$ и непрерывности $F^{-1}(\psi \hat{h})(x)$ следует, что для некоторого $\delta>0$

$$
\Phi\left(\left|F^{-1}(\psi \hat{h})\left(x^{0}\right)\right| / 2 \lambda\right) \geqslant \delta
$$

в некоторой окрестности точки $x^{0}$. Это противоречит $(2)$, так как $F^{-1}(\psi \hat{h})(x)$ не зависит от $x_{j}$.

Из (3) и предположения $\Phi(t)>0, t>0$, вытекает $F^{-1}(\psi \hat{h})(x) \equiv 0$. Отсюда из произвольности $\psi(\xi) \in C_{0}^{\infty}\left(G_{j}\right)$ следует, что распределение $\hat{h}(\xi)$ должно быть сосредоточенным на плоскостях $\xi_{i}=\xi_{j}=0, i, j \in I, i \neq j$.

Положим теперь для $i, j \in I, i \neq j$,

$$
G_{i j}=\left\{\xi \in \mathbb{R}^{n}: \xi_{l} \neq 0, \quad l \in I \backslash\{i, j\}\right\} .
$$

Тогда $G_{i j}$ открыто. Повторяя рассуждения, проведенные для случая $G_{j}$, легко доказать, что распределение $\psi(\xi) \hat{h}(\xi)$ тождественно равно нулю для любой функции $\psi \in C_{0}^{\infty}\left(G_{i j}\right)$. Тем самым мы доказали, что $\hat{h}(\xi)$ сосредоточено на плоскостях $\xi_{i_{1}}=\xi_{i_{2}}=\xi_{i_{3}}=0, i_{1}, i_{2}, i_{3} \in I$.

Повторяя рассуждения, приведенные вьше, еше $k-3$ раз, выводим, что распределение $\hat{h}(\xi)$ сосредоточено в точке $\xi_{1}=\cdots=\xi_{n}=0$, т.е. $h(x)$ - полином, что возможно лишь когда $h(x) \equiv 0$. Это противоречит условию теоремы. Теорема 1 доказана.

Из теоремы 1 и ее доказательства вытекают следующие результаты.

СлЕДСТвИЕ 1. Пусть $\Phi(t)>0, t>0, f \in L_{\Phi}\left(\mathbb{R}^{n}\right), \quad f(x) \not \equiv 0 u \xi^{0} \in \operatorname{sp}(f)$. Тогда существует последовательность точек спектра $\operatorname{sp}(f)$ с ненулевыми компонентами, сходящаяся $к \xi^{0}$.

СЛЕДСТВИЕ 2. Пусть $\Phi(t)>0, \quad t>0, \quad f \in L_{\Phi}\left(\mathbb{R}^{n}\right), \quad f(x) \not \equiv 0 u$ $\xi^{0} \in \operatorname{sp}(f)$ - произвольная точка. Тогда ограничение распределения $\hat{f}(\xi)$ на любую окрестность $\xi^{0}$ также не может быть сосредоточенным на гиперплоскостях $\xi_{j}=\xi_{j}^{0}, \quad j=1, \ldots, n$.

ЗАмечАниЕ 1. Предположение $\Phi(t)>0, t>0$, в утверждении теоремы 1 нельзя снять, ибо в противном случае $L_{\Phi}\left(\mathbb{R}^{n}\right)$ содержит все постоянные функции. 
ЗАмечаниЕ 2 . Пусть $1 \leqslant p<\infty, f(x) \in L_{p}\left(\mathbb{R}^{n}\right), f(x) \not \equiv 0$ и $\operatorname{sp}(f)$ ограничен. Тогда в силу теоремы 1 распределение $\hat{f}(\xi)$ не может быть сосредоточенным на гиперплоскостях $\xi_{j}=\xi_{j}^{0}, j=1, \ldots, n$, где $\xi^{0}$ есть произвольная точка. В то же время $\hat{f}(\xi)$ может быть сосредоточенным на сфере. Действительно, пусть $n=3$ и $f(x)=\frac{\sin |x|}{|x|}$. Тогда, как известно (см. [14]),

$$
\operatorname{sp}(f)=\{\xi:|\xi|=1\}
$$

и, как легко показать, $f(x) \in L_{p}\left(\mathbb{R}^{n}\right)$ для любого $p>3$.

ЗАмечание 3 . Пусть $\Phi(t)>0$ для $t>0, f \in L_{\Phi}\left(\mathbb{R}^{n}\right), f(x) \not \equiv 0$ и $\xi^{0} \in \operatorname{sp}(f)-$ произвольная точка. Тогда с помощью ортогонального преобразования координат можно доказать, что ограничение $\hat{f}(\xi)$ на любую окрестность точки $\xi^{0}$ не может быть сосредоточенным на любом конечном числе плоских гиперплоскостей.

\section{§2. Поведение последовательности норм производных}

Пусть $K$ - некоторое компактное множество в $\mathbb{R}^{n}$ и $\Phi(t)$ - произвольная функция Юнга. Обозначим

$$
\mathfrak{M}_{K \Phi}=\left\{f(x) \in L_{\Phi}\left(\mathbb{R}^{n}\right): \operatorname{supp} F f \subset K\right\}
$$

Исследуем сначала некоторые свойства пространств $\mathfrak{M}_{K \Phi}$.

ЛЕмма 3. Имеют место следующие непрерывнье вложения:

$$
\mathfrak{M}_{K 1} \subset \mathfrak{M}_{K \Phi} \subset \mathfrak{M}_{K \infty}
$$

əдe

$$
\mathfrak{M}_{K p}=\left\{f(x) \in L_{p}\left(\mathbb{R}^{n}\right): \operatorname{supp} F f \subset K\right\}, \quad 1 \leqslant p \leqslant \infty
$$

ДокаЗАТЕльСтво. Пусть $\widehat{\varphi} \in C_{0}^{\infty}\left(\mathbb{R}^{n}\right), \widehat{\varphi}$ равна единице в некоторой окрестности $K$ и $f \in \mathfrak{M}_{K \Phi}$. Тогда $\hat{f}=\hat{\varphi} \hat{f}$. Отсюда и из леммы 1 следует, что

$$
|\varphi * f(x)|=\left|\int \varphi(y) f(x-y) d y\right| \leqslant\|\varphi\|_{\bar{\Phi}}\|f\|_{(\Phi)}
$$

для всех $x \in \mathbb{R}^{n}$. Следовательно,

$$
\|f\|_{\infty}=\|\varphi * f\|_{\infty} \leqslant\|\varphi\|_{\Phi}\|f\|_{(\Phi)} .
$$

Тем самым мы доказали правую часть (4), причем можно выбрать константу

$$
M_{1}=\inf \left\{\|\varphi\|_{\bar{\Phi}}: \widehat{\varphi} \in C_{0}^{\infty}\left(\mathbb{R}^{n}\right), \quad \widehat{\varphi}=1 \text { в некоторой окрестности } K\right\} .
$$

Пусть теперь $g \in \mathfrak{M}_{K 1}$ и $K \subset \Delta_{\nu}$. Тогда из неравенства Никольского следует, что

$$
\|g\|_{\infty} \leqslant 2^{n}\left(\nu_{1} \ldots \nu_{n}\right)\|g\|_{1}
$$


Положим

$$
H=\inf \left\{M>0: \Phi\left(\frac{1}{M}\right) \leqslant \frac{\|g\|_{\infty}}{\|g\|_{1}}\right\} .
$$

Тогда для любого $\epsilon>0$ имеем

$$
\int \Phi\left(\frac{|g(x)|}{\|g\|_{\infty}(H+\epsilon)}\right) d x \leqslant \Phi\left(\frac{1}{H+\epsilon}\right) \frac{\|g\|_{1}}{\|g\|_{\infty}} \leqslant 1,
$$

ибо, очевидно, $\Phi(\lambda t) \leqslant \lambda \Phi(t)$, если $0 \leqslant \lambda \leqslant 1$. Отсюда получим

$$
\|g\|_{(\Phi)} \leqslant H\|g\|_{\infty}
$$

Для доказательства непрерывности вложения выберем такую функцию $\widehat{\varphi} \in$ $C_{0}^{\infty}\left(\mathbb{R}^{n}\right)$, что $\widehat{\varphi}=1$ в некоторой окрестности компакта $K$. Тогда из доказанной правой части (4) вытекает, что

$$
\|g\|_{(\Phi)}=\|g * \varphi\|_{(\Phi)} \leqslant 2\|g\|_{1}\|\varphi\|_{\Phi}<\infty .
$$

Ясно, что $\|\varphi\|_{\Phi}<\infty$. Отсюда константу вложения можно определить следуюшей величиной:

$$
2 \inf \left\{\|\varphi\|_{\Phi}: \widehat{\varphi} \in C_{0}^{\infty}\left(\mathbb{R}^{n}\right), \quad \widehat{\varphi}=1 \text { в некоторой окрестности } K\right\} .
$$

Лемма 3 доказана.

Лемма 4. Пусть $\Phi(t)>0$ для $t>0$. Тогда

$$
\lim _{|x| \rightarrow \infty} f(x)=0
$$

для всех $f \in \mathfrak{M}_{K \Phi}$.

ДоказАтЕЛЬСтво. Пусть $K \subset \Delta_{\nu}$. Докажем методом от противного. Допустим, что существуют функция $f \in \mathfrak{M}_{\nu \Phi}$, константа $c>0$ и последовательность точек $\left|x^{m}\right| \rightarrow \infty$ такие, что

$$
\left|f\left(x^{m}\right)\right| \geqslant 2 c, \quad m=1,2, \ldots
$$

Не теряя общности, можем считать, что

$$
\int_{\mathbb{R}^{n}} \Phi(|f(x)|) d x<\infty
$$

и $\left|x_{1}^{m}\right| \rightarrow \infty, m \rightarrow \infty$. Из леммы 1 , из равенства

$$
f(x)-f\left(x^{m}\right)=\int_{x_{1}^{m}}^{x_{1}} \frac{\partial}{\partial t_{1}} f(t) d t
$$

и неравенства Бернштейна-Никольского [2] вытекает

$$
\left|f(x)-f\left(x^{m}\right)\right| \leqslant \nu_{1}\|f\|_{\infty}\left|x_{1}-x_{1}^{m}\right|
$$


для всех $x \in \mathbb{R}^{n}$ и $m \geqslant 1$.

Положим $r=c / \nu_{1}\|f\|_{\infty}$; получим из $(6),(8)$

$$
|f(x)| \geqslant c \text { для }\left|x_{1}-x_{1}^{m}\right| \leqslant r \text { и } m \geqslant 1 .
$$

С другой стороны, не теряя обшности, можем считать, что

$$
x_{1}^{m+1}-x_{1}^{m} \geqslant r, \quad m \geqslant 1
$$

Следовательно, из (7) и (9) имеем

$$
\begin{gathered}
\infty>\int_{\mathbb{R}^{n}} \Phi(|f(x)|) d x \geqslant \sum_{m=1}^{\infty} \int_{\left|x-x^{m}\right| \leqslant r} \Phi(|f(x)|) d x \\
\geqslant \sum_{m=1}^{\infty} \Phi(c) \operatorname{mes} B\left(x^{m}, r\right)=\sum_{m=1}^{\infty} \pi r^{n} \Phi(c)=\infty
\end{gathered}
$$

где $B\left(x^{m}, r\right)$ есть шар радиуса $r$ с центром в точке $x^{m}$, что невозможно. Лемма 4 доказана.

ЗАмечАниЕ 4 . В случае, когда $\Phi(t)=t^{p}, 1 \leqslant p<\infty$, лемма 4 доказана М. Планшерелем и Г. Пойя (см., например, [2]) другим методом. Лемма 4 неверна, если существует такая точка $t_{0}>0$, что $\Phi\left(t_{0}\right)=0$, ибо в этом случае $\mathfrak{M}_{K \Phi}$ содержит все постоянные функции.

Центральным результатом данного параграфа является решение следующей проблемы. Пусть $f \in L_{\Phi}\left(\mathbb{R}^{n}\right)$ и $\operatorname{sp}(f)$ ограничен. Тогда ясно, что $D^{\alpha} f(x) \in$ $L_{\Phi}\left(\mathbb{R}^{n}\right)$ для всех $\alpha \geqslant 0$. Ставится вопрос о характере поведения последовательности $\left\|D^{\alpha} f\right\|_{(\Phi)}, \alpha \geqslant 0$. Оказывается, что это поведение полностью характеризуется спектром функции $f$. А именно, справедлива следующая

Теорема 2. Пусть $\Phi(t)$ - произвольная функиия Юнга, $f(x) \in L_{\Phi}\left(\mathbb{R}^{n}\right)$ и $\operatorname{sp}(f)$ ограничен. Тогда

$$
\lim _{|\alpha| \rightarrow \infty}\left(\left\|D^{\alpha} f\right\|_{(\Phi)} / \sup _{\operatorname{sp}(f)}\left|\xi^{\alpha}\right|\right)^{1 /|\alpha|}=1
$$

ДокАЗАтЕЛьство. Будем считать, что $f(x) \not \equiv 0$. Докажем сначала, что

$$
\underset{|\alpha| \rightarrow \infty}{\lim _{\mid \rightarrow \infty}}\left(\left\|D^{\alpha} f\right\|_{(\Phi)} /\left|\xi^{\alpha}\right|\right)^{1 /|\alpha|} \geqslant 1
$$

для любой точки $\xi \in \operatorname{sp}(f)$.

Действительно, пусть $\xi^{0} \in \operatorname{sp}(f), \xi_{j}^{0} \neq 0, j=1, \ldots, n$. (В силу теоремы 1 такие точки сушествуют, если $\Phi(t)>0$ для $t>0$.) Для удобства считаем, что $\xi_{j}^{0}>0, j=1, \ldots, n$. Далее, зафиксируем произвольное число $\epsilon>0$ такое, что $2 \epsilon<\xi_{j}^{0}, j=1, \ldots, n$, и область $G\left(\xi^{0} \in G\right)$, содержащуюся в кубе $K=\left\{\xi: \xi_{j}^{0}-\right.$ $\left.\epsilon \leqslant \xi_{j} \leqslant \xi_{j}^{0}+\epsilon, j=1, \ldots, n\right\}$, и выберем функции $\hat{v}(\xi), \widehat{\omega}(\xi) \in C_{0}^{\infty}(G)$, для которых $\xi^{0} \in \operatorname{supp}(\hat{v} \hat{f})$ и $\langle\hat{v} \hat{f}, \widehat{w}\rangle \neq 0$. 
Пусть $\psi \in C_{0}^{\infty}(G)$ и $\psi=1$ в некоторой окрестности supp $\widehat{w}$. Тогда для любого $\alpha \geqslant 0$ имеем

$$
\begin{aligned}
\langle\hat{v} \hat{f}, \widehat{w}\rangle & =\left\langle\psi(\xi) \xi^{-\alpha} \xi^{\alpha} \hat{v}(\xi) \hat{f}(\xi), \widehat{w}(\xi)\right\rangle=\left\langle\xi^{\alpha} \hat{v}(\xi) \hat{f}(\xi), \psi(\xi) \xi^{-\alpha} \widehat{w}(\xi)\right\rangle \\
& =\left\langle F^{-1}\left(\xi^{\alpha} \hat{v}(\xi) \hat{f}(\xi)\right), F\left(\psi(\xi) \xi^{-\alpha} \widehat{w}(\xi)\right)\right\rangle \\
& =\left\langle F^{-1}\left(\xi^{\alpha} \hat{v}(\xi) \hat{f}(\xi)\right), F\left(\xi^{-\alpha} \widehat{w}(\xi)\right)\right\rangle .
\end{aligned}
$$

Следовательно, в силу лемм 1,2 имеем

$$
0<|\langle\hat{v} \hat{f}, \widehat{w}\rangle|=\left|\left\langle D^{\alpha}(v * f), F \widehat{w}_{\alpha}\right\rangle\right| \leqslant 2\|v\|_{1}\left\|D^{\alpha} f\right\|_{(\Phi)}\left\|F \widehat{w}_{\alpha}\right\|_{(\bar{\Phi})},
$$

где $\widehat{w}_{\alpha}(\xi)=\xi^{-\alpha} \widehat{w}(\xi), \alpha \geqslant 0$. Докажем теперь, что

$$
\left\|F \widehat{w}_{\alpha}\right\|_{(\bar{\Phi})} \leqslant C\left(\xi^{0}-2 \epsilon\right)^{-\alpha}, \quad \alpha \geqslant 0
$$

где $C$ - некоторая константа, не зависящая от $\alpha$, и $\xi^{0}-2 \epsilon=\left(\xi_{1}^{0}-2 \epsilon, \ldots, \xi_{n}^{0}-2 \epsilon\right)$.

В самом деле, имеем для любых $\alpha, \beta \in \mathbb{Z}_{+}^{n}$ и $x \in \mathbb{R}^{n}$

$$
\begin{aligned}
(-i)^{|\beta|} x^{\beta} F \widehat{\omega}_{\alpha}(x) & =(-i)^{|\beta|} \int_{G} x^{\beta} e^{-i x \xi} \xi^{-\alpha} \widehat{\omega}(\xi) d \xi=\int_{G}\left(D_{\xi}^{\beta} e^{-i x \xi}\right)\left(\xi^{-\alpha} \widehat{\omega}(\xi)\right) d \xi \\
& =(-1)^{|\beta|} \int_{G} e^{-i x \xi} D^{\beta}\left(\xi^{-\alpha} \widehat{\omega}(\xi)\right) d \xi
\end{aligned}
$$

Отсюда, учитывая формулу Лейбница и определение области $G$, получим для $|\beta| \leqslant 2 n$

$$
\begin{aligned}
\sup _{x \in \mathbb{R}^{n}} & \left|x^{\beta} F \widehat{\omega}_{\alpha}(x)\right| \\
& \leqslant \sum_{\gamma \leqslant \beta}\left\{\frac{\beta !}{\gamma !(\beta-\gamma) !} \prod_{k=1}^{n} \alpha_{k} \ldots\left(\alpha_{k}+\gamma_{k}-1\right) \int_{G}\left|\xi^{-(\alpha+\gamma)} D^{\beta-\gamma} \widehat{\omega}(\xi)\right| d \xi\right\} \\
& \leqslant C_{1}\left(\xi^{0}-\epsilon\right)^{-\alpha} \sum_{\gamma \leqslant \beta} \frac{\beta !}{\gamma !(\beta-\gamma) !} \prod_{k=1}^{n} \alpha_{k} \ldots\left(\alpha_{k}+\gamma_{k}-1\right)
\end{aligned}
$$

где

$$
C_{1}=\max \left\{\int_{G}\left|\xi^{-\gamma} D^{\nu-\gamma} \widehat{\omega}(\xi)\right| d \xi: \gamma \leqslant \nu, \quad|\nu| \leqslant 2 n\right\}<\infty .
$$

Из (14) вытекает, что

$$
\sup _{x \in \mathbb{R}^{n}}\left|x^{\beta} \widehat{\omega}_{\alpha}(x)\right| \leqslant C_{2}\left(\xi^{0}-2 \epsilon\right)^{-\alpha}
$$

для всех $|\beta| \leqslant 2 n$ и $\alpha \geqslant 0$. Отсюда получим константу $C_{3}$ такую, что для всех $\alpha \geqslant 0$

$$
\sup _{x \in \mathbb{R}^{n}}\left(1+x_{1}^{2}\right) \ldots\left(1+x_{n}^{2}\right)\left|F \widehat{\omega}_{\alpha}(x)\right| \leqslant C_{3}\left(\xi^{0}-2 \epsilon\right)^{-\alpha} .
$$


Далее, пусть число $0<\lambda_{0}<\infty$ такое, что $\bar{\Phi}\left(C_{3} / \lambda_{0}\right) \leqslant \pi^{-n}$. Тогда из (15), монотонности $\bar{\Phi}(t)$ и $\bar{\Phi}(\lambda t) \leqslant \lambda \bar{\Phi}(t), 0 \leqslant \lambda \leqslant 1$, вытекает

$$
\begin{aligned}
\int \bar{\Phi}\left(\frac{\left|F \widehat{\omega}_{\alpha}\right|}{\lambda_{0}\left(\xi^{0}-2 \epsilon\right)^{-\alpha}}\right) d x & \leqslant \int \bar{\Phi}\left(\frac{C_{3}}{\lambda_{0}\left(1+x_{1}^{2}\right) \ldots\left(1+x_{n}^{2}\right)}\right) d x \\
& \leqslant \bar{\Phi}\left(\frac{C_{3}}{\lambda_{0}}\right) \int \frac{d x}{\left(1+x_{1}^{2}\right) \ldots\left(1+x_{n}^{2}\right)} \leqslant 1 .
\end{aligned}
$$

Тогда по определению

$$
\left\|F \widehat{\omega}_{\alpha}\right\|_{(\bar{\Phi})} \leqslant \lambda_{0}\left(\xi^{0}-2 \epsilon\right)^{-\alpha}
$$

для всех $\alpha \geqslant 0$. Тем самым мы доказали (13).

Комбинируя (12) и (13), выводим

$$
1 \leqslant \lim _{|\alpha| \rightarrow \infty}\left(\left(\xi^{0}-2 \epsilon\right)^{-\alpha}\left\|D^{\alpha} f\right\|_{(\Phi)}\right)^{1 /|\alpha|} .
$$

Отсюда, учитывая произвольность $\epsilon>0$ и

$$
\left(\frac{\left(\xi^{0}-2 \epsilon\right)^{-\alpha}}{\left(\xi^{0}\right)^{-\alpha}}\right)^{1 /|\alpha|} \leqslant \max _{1 \leqslant j \leqslant n} \frac{\xi_{j}^{0}}{\xi_{j}^{0}-2 \epsilon},
$$

немедленно получим (11).

Покажем теперь (11) для "нулевых" точек. Пусть $\xi^{0} \in \operatorname{sp}(f), \quad \xi^{0} \neq 0$ и $\xi_{1}^{0} \ldots \xi_{n}^{0}=0$. Для удобства считаем, что $\xi_{j}^{0}>0, j=1, \ldots, k$, и $\xi_{k+1}^{0}=\cdots=\xi_{n}^{0}=0$ $(1 \leqslant k<n)$. Заметим, что нам достаточно доказать (11) только для таких $\alpha$, что $\alpha_{k+1}=\cdots=\alpha_{n}=0$. И тогда доказательство проводится совершенно аналогично тому, как это было сделано выше после единственной модификации выбора $\epsilon$. Зафиксируем произвольное число $\epsilon>0$ такое, что $2 \epsilon<\min _{1 \leqslant j \leqslant k} \xi_{j}^{0}$, и область $G$ $\left(\xi^{0} \in G\right)$, содержашуюся в кубе

$$
K=\left\{\xi: \xi_{j}^{0}-\epsilon \leqslant \xi_{j} \leqslant \xi_{j}^{0}+\epsilon, \quad j=1, \ldots, n\right\} .
$$

Докажем теперь (10) для случая, когда $\Phi(t)>0, t>0$. Докажем сначала методом от противного, что

$$
\varliminf_{|\alpha| \rightarrow \infty}\left(\left\|D^{\alpha} f\right\|_{(\Phi)} / \sup _{\operatorname{sp}(f)}\left|\xi^{\alpha}\right|\right)^{1 /|\alpha|} \geqslant 1 .
$$

Допустим, что сушествует подпоследовательность $I_{1}$ такая, что

$$
\text { (I }) \lim _{|\alpha| \rightarrow \infty}\left(\left\|D^{\alpha} f\right\|_{(\Phi)} / \sup _{\operatorname{sp}(f)}\left|\xi^{\alpha}\right|\right)^{1 /|\alpha|}<1,
$$

где символ $\left(I_{1}\right)$ означает, что мы берем предел только для мультииндексов $\alpha \in I_{1}$. Тогда сушествуют подпоследовательность $I_{2} \subset I_{1}$ и числа $0 \leqslant \beta_{j} \leqslant 1, j=$ $1, \ldots, n$, такие, что $|\beta|=1$ и

$$
\left(I_{2}\right) \lim _{|\alpha| \rightarrow \infty} \frac{\alpha_{j}}{|\alpha|}=\beta_{j}, \quad j=1, \ldots, n .
$$


Покажем теперь

$$
\lim _{\gamma \rightarrow \beta} \sup _{\operatorname{sp}(f)}\left|\xi^{\gamma}\right|=\sup _{\operatorname{sp}(f)}\left|\xi^{\beta}\right|
$$

если $\gamma \in \mathbb{R}_{+}^{n}$ и $\gamma \rightarrow \beta$.

Зафиксируем $h>1$. Тогда существует такое число $\epsilon>0$, что $h \gamma \geqslant \beta$ для $\gamma \in \mathbb{R}_{+}^{n}$ и $|\gamma-\beta| \leqslant \epsilon$. Далее, пусть $|\xi| \leqslant M$ для всех $\xi \in \operatorname{sp}(f)$. Тогда для $\xi \in \operatorname{sp}(f)$ и $\gamma \in \mathbb{R}_{+}^{n},|\gamma-\beta| \leqslant \epsilon$, имеем

$$
\left|\xi^{\gamma}\right|=\left|\xi^{\gamma-\beta / h}\right|\left|\xi^{\beta}\right|^{1 / h} \leqslant M^{|\gamma-\beta / h|} \sup _{\operatorname{sp}(f)}\left|\xi^{\beta}\right|^{1 / h} .
$$

Следовательно,

$$
\varlimsup_{\gamma \rightarrow \beta} \sup _{\operatorname{sp}(f)}\left|\xi^{\gamma}\right| \leqslant M^{|\beta|(1-1 / h)} \sup _{\operatorname{sp}(f)}\left|\xi^{\beta}\right|^{1 / h} .
$$

Устремляя $h$ к 1 , выводим

$$
\varlimsup_{\gamma \rightarrow \beta} \sup _{\operatorname{sp}(f)}\left|\xi^{\gamma}\right| \leqslant \sup _{\operatorname{sp}(f)}\left|\xi^{\beta}\right| .
$$

Для получения (19) нам осталось показать

$$
\underset{\gamma \rightarrow \beta}{\lim _{\operatorname{sp}(f)}} \sup \left|\xi^{\gamma}\right| \geqslant \sup _{\operatorname{sp}(f)}\left|\xi^{\beta}\right| .
$$

Пусть точка $\xi^{*} \in \operatorname{sp}(f)$ такая, что $\left|\xi^{* \beta}\right|=\sup _{\mathrm{sp}(f)}\left|\xi^{\beta}\right|$. Тогда в силу теоремы 1 распределение $\hat{f}(\xi)$ не сосредоточено на гиперплоскостях $\xi_{j}=0, j=1, \ldots, n$. Следовательно, $\left|\xi^{* \beta}\right|>0$. Далее, ограничение распределения $\hat{f}(\xi)$ на любую окрестность точки $\xi^{*}$ также не может быть сосредоточенным на гиперплоскостях $\xi_{j}=0, j=1, \ldots, n$. Следовательно, существует последовательность точек $m \xi \in \operatorname{sp}(f), m \geqslant 1$, таких, что $m \xi_{j} \neq 0, j=1, \ldots, n$, для любого $m \geqslant 1$ и $m \xi \rightarrow \xi^{*}, m \rightarrow \infty$. Тогда

$$
\sup _{\operatorname{sp}(f)}\left|\xi^{\gamma}\right| \geqslant\left|{ }_{m} \xi^{\gamma}\right|
$$

для любого $m \geqslant 1$. Отсюда

$$
\varliminf_{\gamma \rightarrow \beta} \sup _{\operatorname{sp}(f)}\left|\xi^{\gamma}\right| \geqslant \varliminf_{\gamma \rightarrow \beta}\left|m \xi^{\gamma}\right|=\left|m \xi^{\beta}\right| .
$$

Устремляя $m$ к $\infty$, выводим (20), а следовательно, и (19).

Далее, пусть $\lambda>1$. Тогда существует число $k \geqslant 1$ такое, что $\left.\lambda\right|_{k} \xi^{\beta}|\geqslant| \xi^{* \beta} \mid$. Следовательно, из (18), (19) и (11) вытекает

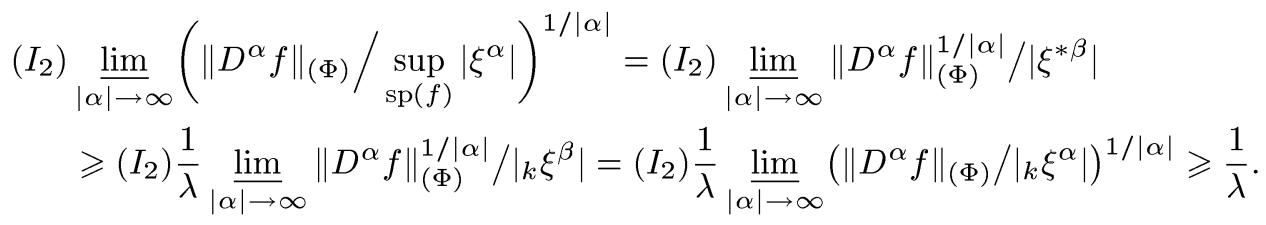

Это противоречит (17) при $\lambda \rightarrow 1$. Тем самым мы доказали (16). 
Наконец, покажем

$$
\varlimsup_{|\alpha| \rightarrow \infty}\left(\left\|D^{\alpha} f\right\|_{(\Phi)} / \sup _{\operatorname{sp}(f)}\left|\xi^{\alpha}\right|\right)^{1 /|\alpha|} \leqslant 1
$$

Зафиксируем область $G \supset \operatorname{sp}(f)$ и функцию $\psi \in C_{0}^{\infty}(G)$ такие, что $\psi(\xi)$ равна единице в некоторой окрестности $\operatorname{sp}(f)$. Положим $h_{\alpha}(\xi)=\psi(\xi) \xi^{\alpha}, \alpha \geqslant 0$. Тогда из неравенства Гёльдера следует, что для любого $s>n / 2$

$$
\begin{aligned}
\left\|F^{-1} h_{\alpha}\right\|_{1} & =\int\left(\left|\hat{h}_{\alpha}(\xi)\right|^{2}\right)^{1 / 2} d \xi \\
& \leqslant\left(\int\left|\hat{h}_{\alpha}(\xi)\right|^{2}\left(1+|\xi|^{2}\right)^{s} d \xi\right)^{1 / 2}\left(\int\left(1+|\xi|^{2}\right)^{-s} d \xi\right)^{1 / 2} .
\end{aligned}
$$

Следовательно,

$$
\left\|F^{-1} h_{\alpha}\right\|_{1} \leqslant C^{\prime}\left\|h_{\alpha}\right\|_{(s)}
$$

где $C^{\prime}=C^{\prime}(s)$ не зависит от $h_{\alpha}$.

Комбинируя $(22)$, топологическое равенство $H_{(k)}=W_{k, 2}\left(\mathbb{R}^{n}\right)$ и

$$
\left\|D^{\alpha} f\right\|_{(\Phi)}=\left\|F^{-1}\left(\psi(\xi) \xi^{\alpha}\right) * f\right\|_{(\Phi)} \leqslant 2\left\|F^{-1}\left(\psi(\xi) \xi^{\alpha}\right)\right\|_{1}\|f\|_{(\Phi)}
$$

получим

$$
\left\|D^{\alpha} f\right\|_{(\Phi)} \leqslant C\left\|\psi(\xi) \xi^{\alpha}\right\|_{k, 2}\|f\|_{(\Phi)}, \quad \alpha \geqslant 0
$$

где $C$ не зависит от $f$ и $\alpha$ и $k=\left[\frac{n}{2}\right]+1$.

Из формулы Лейбница получим константу $C_{1}=C_{1}(\psi, k)$ такую, что

$$
\left\|\psi(\xi) \xi^{\alpha}\right\|_{k, 2} \leqslant C_{1}|\alpha|^{k} \sup \left\{\sup _{G}\left|\xi^{\alpha-\gamma}\right|: \gamma \leqslant \alpha, \quad|\gamma| \leqslant k\right\}, \quad \alpha \geqslant 0
$$

С другой стороны, имеем

$$
\lim _{|\alpha| \rightarrow \infty}\left(\sup \left\{\sup _{G}\left|\xi^{\alpha-\gamma}\right|: \gamma \leqslant \alpha, \quad|\gamma| \leqslant k\right\}\right)^{1 /|\alpha|} / \sup _{G}\left|\xi^{\alpha}\right|^{1 /|\alpha|}=1 .
$$

Докажем (25) методом от противного. Допустим, что существуют последовательность $I_{1}$ и число $\delta>1$ такие, что

$$
\sup \left\{\sup _{G}\left|\xi^{\alpha-\gamma}\right|^{1 /|\alpha|}: \gamma \leqslant \alpha, \quad|\gamma| \leqslant k\right\} \geqslant \delta \sup _{G}\left|\xi^{\alpha}\right|^{1 /|\alpha|}, \quad \alpha \in I_{1} .
$$

Следовательно, существуют подпоследовательность $I_{2} \subset I_{1}$, числа $0 \leqslant \beta_{j} \leqslant 1$, $j=1, \ldots, n$, и мультииндекс $\gamma^{0},\left|\gamma^{0}\right| \leqslant k$, такие, что $|\beta|=1$ и

$$
\begin{gathered}
\left(I_{2}\right) \lim _{|\alpha| \rightarrow \infty} \frac{\alpha_{j}-\gamma_{j}^{0}}{|\alpha|}=\beta_{j}, \quad j=1, \ldots, n, \\
\sup \left\{\sup _{G}\left|\xi^{\alpha-\gamma}\right|^{1 /|\alpha|}: \gamma \leqslant \alpha, \quad|\gamma| \leqslant k\right\}=\sup _{G}\left|\xi^{\alpha-\gamma^{0}}\right|^{1 /|\alpha|}
\end{gathered}
$$


для всех $\alpha \in I_{2}$. Следовательно, рассуждениями, аналогичными проведенным в доказательстве (19), получим

$$
\left(I_{2}\right) \lim _{|\alpha| \rightarrow \infty} \sup _{G}\left|\xi^{\alpha-\gamma^{0}}\right|^{1 /|\alpha|}=\left(I_{2}\right) \lim _{|\alpha| \rightarrow \infty} \sup _{G}\left|\xi^{\alpha}\right|^{1 /|\alpha|}=\sup _{G}\left|\xi^{\beta}\right|>0
$$

что противоречит (26). Равенство (25) доказано.

Комбинируя (23)-(25), получим

$$
\varlimsup_{|\alpha| \rightarrow \infty}\left\|D^{\alpha} f\right\|_{(\Phi)}^{1 /|\alpha|} / \sup _{G}\left|\xi^{\alpha}\right|^{1 /|\alpha|} \leqslant 1
$$

Сейчас мы допустим (от противного), что (21) не выполняется. Тогда существуют подпоследовательность $J$ и числа $\lambda>1,0 \leqslant \beta_{j} \leqslant 1, j=1, \ldots, n$, такие, что $|\beta|=1$ и

$$
\begin{aligned}
& \text { (J) } \lim _{|\alpha| \rightarrow \infty}\left\|D^{\alpha} f\right\|_{(\Phi)}^{1 /|\alpha|} / \sup _{\operatorname{sp}(f)}\left|\xi^{\alpha}\right|^{1 /|\alpha|}=\lambda, \\
& \text { (J) } \lim _{|\alpha| \rightarrow \infty} \frac{\alpha_{j}}{|\alpha|}=\beta_{j}, \quad j=1, \ldots, n .
\end{aligned}
$$

Следовательно, из справедливости (19) при замене $\operatorname{sp}(f)$ множеством $G$ (доказательство этого проводится аналогично, так как $G$ открыто) и (27) получим

$$
\sup _{G}\left|\xi^{\beta}\right| / \sup _{\operatorname{sp}(f)}\left|\xi^{\beta}\right| \geqslant \lambda
$$

для любой области $G \supset \operatorname{sp}(f)$, что невозможно, так как $\sup _{\mathrm{sp}(f)}\left|\xi^{\beta}\right|>0$. Доказательство первого случая закончено.

Рассмотрим теперь оставшийся случай, т.е. когда $\Phi\left(t_{0}\right)=0$ для некоторой точки $t_{0}>0$. Этот случай оказывается более трудным. Отметим, что многие факты, используемые в доказательстве предыдушего случая, здесь неверны (например, равенство (19)).

Докажем сначала, что если $\sup _{\mathrm{sp}(f)}\left|\xi^{\alpha}\right|=0$, то $D^{\alpha} f(x) \equiv 0$ (для того же $\alpha$ ). В самом деле, не теряя общности, можно считать, что $\alpha_{j} \neq 0, j=1, \ldots, k$, и $\alpha_{k+1}=\cdots=\alpha_{n}=0(1 \leqslant k \leqslant n)$. Тогда распределение $\hat{f}(\xi)$ сосредоточено на $\xi_{j}=0, j \in\{1, \ldots, k\}=I$. Нам достаточно доказать случай $\alpha_{1}=\cdots=\alpha_{k}=1$.

Докажем теперь, что если $\xi^{\alpha} \psi(\xi) \hat{f}(\xi)$ сосредоточено на плоскости $\xi_{i_{1}}=\ldots$ $\cdots=\xi_{i_{l}}=0$ для некоторых $i_{1}, \ldots, i_{l} \in I$ и $\psi \in C_{0}^{\infty}\left(\mathbb{R}^{n}\right)$, то $D^{\alpha} F^{-1} \psi * f(x) \equiv 0$. Действительно, $\xi^{\alpha} \psi(\xi) \hat{f}(\xi)$ сосредоточено на плоскости $\xi_{1}=\cdots=\xi_{l}=0$ (для краткости считаем, что $\left.i_{j}=j, j=1, \ldots, l\right)$. Следовательно, учитывая одно замечание о теореме 2.3.5, упомянутое в примере 5.1 .2 из [12], получим

$$
F^{-1}\left(\xi^{\alpha} \psi(\xi) \hat{f}(\xi)\right)(x)=\sum_{|\beta| \leqslant N} g_{\beta}\left(x^{\prime \prime}\right)\left(-i x^{\prime}\right)^{\beta}
$$

где $N$ - порядок распределения $\hat{f}(\xi)(N<\infty$, так как supp $\hat{f}$ компактно), $x^{\prime}=\left(x_{1}, \ldots, x_{l}\right), x=\left(x^{\prime}, x^{\prime \prime}\right), \quad \beta \in \mathbb{Z}_{+}^{l}$ и $\hat{g}_{\beta}\left(\xi_{l+1}, \ldots, \xi_{n}\right),|\beta| \leqslant N,-$ pacпределения с компактным носителем. 
С другой стороны, из лемм 1,3 следует

$\left\|F^{-1}\left(\xi^{\alpha} \psi(\xi) \hat{f}(\xi)\right)\right\|_{\infty}=\left\|F^{-1}\left(\xi^{\alpha} \psi(\xi)\right) * f\right\|_{\infty} \leqslant\left\|F^{-1}\left(\xi^{\alpha} \psi(\xi)\right)\right\|_{1}\|f\|_{\infty}<\infty$.

Следовательно, из (28) имеем

$$
F^{-1}\left(\xi^{\alpha} \psi(\xi) \hat{f}(\xi)\right)(x)=D^{\alpha} F^{-1} \psi * f(x)=g_{0}\left(x^{\prime \prime}\right) .
$$

Пусть $\gamma_{1}=0, \gamma_{2}=\cdots=\gamma_{k}=1, \gamma_{k+1}=\cdots=\gamma_{n}=0$. Тогда

$$
D_{x_{1}} D^{\gamma} F^{-1} \psi * f(x)=g_{0}\left(x^{\prime \prime}\right)
$$

Следовательно,

$$
D^{\gamma} F^{-1} \psi * f(x)=i x_{1} g_{0}\left(x^{\prime \prime}\right)+t\left(x_{2}, \ldots, x_{n}\right)
$$

Отсюда, учитьвая $D^{\gamma} F^{-1} \psi * f \in L_{\infty}$ (очевидно), получим $g_{0}\left(x^{\prime \prime}\right) \equiv 0$, т.е.

$$
D^{\alpha} F^{-1} \psi * f(x) \equiv 0
$$

Покажем, далее, что $\xi^{\alpha} \hat{f}(\xi)$ сосредоточено на плоскости $\xi_{1}=\cdots=\xi_{k}=0$. Действительно, для каждого $j \in I$ положим

$$
G_{j}=\left\{\xi \in \mathbb{R}^{n}: \xi_{i} \neq 0, \quad i \in I \backslash\{j\}\right\} .
$$

Тогда $G_{j}$ открыто. Для каждой $\varphi \in C_{0}^{\infty}\left(G_{j}\right)$ выберем функцию $\psi(\xi)$ из $C_{0}^{\infty}\left(G_{j}\right)$ такую, что $\psi=1$ в некоторой окрестности $\operatorname{supp} \varphi$. Тогда $\psi(\xi) \hat{f}(\xi)$ сосредоточено на гиперплоскости $\xi_{j}=0$, и из доказанного утверждения получим

$$
\left\langle\xi^{\alpha} \hat{f}(\xi), \varphi(\xi)\right\rangle=\left\langle\xi^{\alpha} \psi(\xi) \hat{f}(\xi), \varphi(\xi)\right\rangle=\left\langle D^{\alpha} F^{-1} \psi * f, \widehat{\varphi}\right\rangle=0
$$

Тем самым мы доказали, что $\xi^{\alpha} \hat{f}(\xi)$ сосредоточено на плоскостях $\xi_{i}=\xi_{j}=0$, $i, j \in I$.

Положим для $i, j \in I$

$$
G_{i j}=\left\{\xi \in \mathbb{R}^{n}: \xi_{l} \neq 0, \quad l \in I \backslash\{i, j\}\right\} .
$$

Тогда $G_{i j}$ открыто. Повторяя рассуждения, проведенные для случая $G_{j}$, легко доказать, что

$$
\left\langle\xi^{\alpha} \hat{f}(\xi), \varphi(\xi)\right\rangle=0 \quad \forall \varphi \in C_{0}^{\infty}\left(G_{i j}\right)
$$

Тем самым мы доказали, что $\xi^{\alpha} \hat{f}(\xi)$ сосредоточено на плоскостях $\xi_{i_{1}}=\xi_{i_{2}}=$ $\xi_{i_{3}}=0, i_{1}, i_{2}, i_{3} \in I$.

Повторяя рассуждения, приведенные выше, еше $k-3$ раз, выводим, что распределение $\xi^{\alpha} \hat{f}(\xi)$ сосредоточено на плоскости $\xi_{1}=\cdots=\xi_{k}=0$.

Пусть $\psi \in C_{0}^{\infty}\left(\mathbb{R}^{n}\right)$ и $\psi=1$ в некоторой окрестности $\operatorname{sp}(f)$. Тогда для любой $\varphi \in C_{0}^{\infty}\left(\mathbb{R}^{n}\right)$ имеем

$$
\begin{aligned}
\left\langle D^{\alpha} f, \widehat{\varphi}\right\rangle & =\left\langle\xi^{\alpha} \hat{f}(\xi), \varphi(\xi)\right\rangle=\left\langle\xi^{\alpha} \psi(\xi) \hat{f}(\xi), \varphi(\xi)\right\rangle \\
& =\left\langle D^{\alpha} F^{-1} \psi * f, \widehat{\varphi}\right\rangle=\langle 0, \widehat{\varphi}\rangle=0 .
\end{aligned}
$$


С другой стороны, как известно, $F\left(C_{0}^{\infty}\left(\mathbb{R}^{n}\right)\right)$ плотно в $L_{1}\left(\mathbb{R}^{n}\right)$. Следовательно, из последних равенств и $D^{\alpha} f \in L_{\infty}\left(\mathbb{R}^{n}\right)$ получим $D^{\alpha} f(x) \equiv 0$.

Из доказанного следует, что нам достаточно доказать (10) только для таких мультииндексов $\alpha \geqslant 0$, что $\sup _{\operatorname{sp}(f)}\left|\xi^{\alpha}\right|>0$. Обозначим через $P$ множество таких мультииндексов.

Докажем теперь

$$
\text { (P) } \varliminf_{|\alpha| \rightarrow \infty}\left(\left\|D^{\alpha} f\right\|_{(\Phi)} / \sup _{\operatorname{sp}(f)}\left|\xi^{\alpha}\right|\right)^{1 /|\alpha|} \geqslant 1
$$

методом от противного. Допустим, что сушествуют подпоследовательность $I \subset P$, число $\lambda<1$ и вектор $\beta \geqslant 0,|\beta|=1$, такие, что

$$
\begin{aligned}
& \text { (I) } \lim _{|\alpha| \rightarrow \infty}\left(\left\|D^{\alpha} f\right\|_{(\Phi)} / \sup _{\operatorname{sp}(f)}\left|\xi^{\alpha}\right|\right)^{1 /|\alpha|}<\lambda, \\
& \text { (I) } \lim _{|\alpha| \rightarrow \infty} \frac{\alpha}{|\alpha|}=\beta .
\end{aligned}
$$

Отметим, что

$$
\text { (I) } \lim _{|\alpha| \rightarrow \infty} \sup _{\operatorname{sp}(f)}\left|\xi^{\alpha}\right|^{1 /|\alpha|}>0 .
$$

Действительно, в противном случае сушествует такая подпоследовательность $J \subset I$, что

$$
(J) \lim _{|\alpha| \rightarrow \infty} \sup _{\operatorname{sp}(f)}\left|\xi^{\alpha}\right|^{1 /|\alpha|}=0 .
$$

Для любых $1 \leqslant k \leqslant n$ и $i_{1}, \ldots, i_{k} \in\{1, \ldots, n\}$ положим

$$
T_{i_{1} \ldots i_{k}}=\left\{\alpha \geqslant 0: \alpha_{i_{1}} \neq 0, \ldots, \alpha_{i_{k}} \neq 0 \text { и } \alpha_{j}=0, \text { если } j \notin\left\{i_{1}, \ldots, i_{k}\right\}\right\} .
$$

Тогда существуют $1 \leqslant k \leqslant n$ и $i_{1}, \ldots, i_{k} \in\{1, \ldots, n\}$ такие, что $J_{i_{1} \ldots i_{k}}=J \cap$ $T_{i_{1} \ldots i_{k}}$ не ограничено. Следовательно ясно, что

$$
\left(J_{i_{1} \ldots i_{k}}\right) \lim _{|\alpha| \rightarrow \infty} \sup _{\mid \alpha p(f)}\left|\xi^{\alpha}\right|^{1 /|\alpha|} \geqslant\left(J_{i_{1} \ldots i_{k}}\right) \lim _{|\alpha| \rightarrow \infty}\left|\eta^{\alpha}\right|^{1 /|\alpha|}>0
$$

где $\eta$ есть такая произвольная точка $\operatorname{sp}(f)$, что $\eta_{i_{1}} \neq 0, \ldots, \eta_{i_{k}} \neq 0$. А это противоречит (33). Неравенство (32) доказано.

Далее, пусть $\alpha \xi \in \operatorname{sp}(f):\left|{ }_{\alpha} \xi^{\alpha}\right|=\sup _{\operatorname{sp}(f)}\left|\xi^{\alpha}\right|$. Тогда ${ }_{\alpha} \xi_{i_{1}} \neq 0, \ldots, \alpha \xi_{i_{k}} \neq 0$ для любого $\alpha \in J_{i_{1} \ldots i_{k}}$, и путем выбора подпоследовательности, не теряя обшности, можно считать, что для некоторой точки $\xi^{*} \in \mathrm{sp}(f)$

$$
\left(J_{i_{1} \ldots i_{k}}\right) \lim _{|\alpha| \rightarrow \infty} \alpha \xi=\xi^{*} .
$$

Рассмотрим теперь два случая для точки $\xi^{*}$.

Если $\xi_{i_{j}}^{*} \neq 0, j=1, \ldots, k$, то, очевидно,

$$
\left(J_{i_{1} \ldots i_{k}}\right) \lim _{|\alpha| \rightarrow \infty}\left|\alpha \xi^{\alpha}\right|^{1 /|\alpha|}=\left|\xi^{* \beta}\right|=\left(J_{i_{1} \ldots i_{k}}\right) \lim _{|\alpha| \rightarrow \infty}\left|\xi^{* \alpha}\right|^{1 /|\alpha|},
$$


что вместе с $\xi^{*} \in \operatorname{sp}(f),(11)$ и (30) дает нам

$$
\begin{aligned}
1 & \leqslant\left(J_{i_{1} \ldots i_{k}}\right) \lim _{|\alpha| \rightarrow \infty}\left(\left\|D^{\alpha} f\right\|_{(\Phi)} /\left|\xi^{* \alpha}\right|\right)^{1 /|\alpha|} \\
& =\left(J_{i_{1} \ldots i_{k}}\right) \lim _{|\alpha| \rightarrow \infty}\left(\left\|D^{\alpha} f\right\|_{(\Phi)} / \sup _{\operatorname{sp}(f)}\left|\xi^{\alpha}\right|\right)^{1 /|\alpha|}<\lambda<1
\end{aligned}
$$

что невозможно.

В другом случае, не теряя обшности, можно считать, что $\xi_{i_{1}}^{*}=\cdots=\xi_{i_{m}}^{*}=0$ и $\xi_{i_{m+1}}^{*} \neq 0, \ldots, \xi_{i_{k}}^{*} \neq 0$ для некоторого $1 \leqslant m \leqslant k$.

Из (32) и (34) следует, что $\xi^{*} \neq 0$. Следовательно, $m<k$. Далее, учитывая (31), (32), (34), определение ${ }_{\alpha} \xi$ и $\xi_{i_{1}}^{*}=\cdots=\xi_{i_{m}}^{*}=0$, получим $\beta_{i_{1}}=\cdots$ $\cdots=\beta_{i_{m}}=0$. Из равенств

$$
\begin{aligned}
& \left(J_{i_{1} \ldots i_{k}}\right) \lim _{|\alpha| \rightarrow \infty}\left|\alpha \xi_{i_{m+1}}^{\alpha_{i_{m}+1}} \ldots \alpha \xi_{i_{k}}^{\alpha_{i_{k}}}\right|^{1 /|\alpha|}=\left|\xi_{i_{m+1}^{*}}^{{ }^{\beta_{i_{m+1}}}} \ldots \xi_{i_{k}}^{*}{ }^{\beta_{i_{k}}}\right| \\
& =\left.\left(J_{i_{1} \ldots i_{k}}\right) \lim _{|\alpha| \rightarrow \infty}\left|\xi_{i_{m+1}^{*}}^{\alpha^{\alpha_{i}}} \ldots \xi_{i_{k}}^{*}\right|^{\alpha_{i_{k}}}\right|^{1 /|\alpha|},
\end{aligned}
$$

которые очевидны, вытекает что существуют $\nu \in J_{i_{1} \ldots i_{k}}$ и $N>0$ такие, что

$$
\left|\alpha \xi_{i_{l}}\right| \leqslant\left.\lambda^{-1}\right|_{\nu} \xi_{i_{l}} \mid, \quad l=m+1, \ldots, k
$$

для всех $|\alpha| \geqslant N, \alpha \in J_{i_{1} \ldots i_{k}}$.

С другой стороны, из ${ }_{\nu} \xi_{i_{1}} \neq 0, \ldots, \nu \xi_{i_{k}} \neq 0$ и

$$
\left(J_{i_{1} \ldots i_{k}}\right) \lim _{|\alpha| \rightarrow \infty} \alpha \xi_{i_{j}}=\xi_{i_{j}}^{*}=0, \quad j=1, \ldots, m
$$

следует, что существует $M>0$ такое, что

$$
\left|{ }_{\alpha} \xi_{i_{j}}\right| \leqslant\left|{ }_{\nu} \xi_{i_{j}}\right|, \quad j=1, \ldots, m
$$

для всех $|\alpha| \geqslant M, \alpha \in J_{i_{1} \ldots i_{k}}$. Это вместе с (35) дает

$$
{ }_{\alpha} \xi_{i_{j}}\left|\leqslant \lambda^{-1}\right| \nu \xi_{i_{j}} \mid, \quad j=1, \ldots, k,
$$

для всех $|\alpha| \geqslant \max \{M, N\}, \alpha \in J_{i_{1} \ldots i_{k}}$. Следовательно,

$$
\sup _{\operatorname{sp}(f)}\left|\xi^{\alpha}\right|^{1 /|\alpha|}=\left.\left.\right|_{\alpha} \xi^{\alpha}\right|^{1 /|\alpha|} \leqslant\left.\left.\lambda^{-1}\right|_{\nu} \xi^{\alpha}\right|^{1 /|\alpha|}
$$

что вместе с (11) и (30) дает

$$
\begin{aligned}
1 & \leqslant\left(J_{i_{1} \ldots i_{k}}\right) \underset{|\alpha| \rightarrow \infty}{\lim }\left(\left\|D^{\alpha} f\right\|_{(\Phi)} /\left.\right|_{\nu} \xi^{\alpha} \mid\right)^{1 /|\alpha|} \\
& \leqslant\left(J_{i_{1} \ldots i_{k}}\right) \lambda^{-1} \lim _{|\alpha| \rightarrow \infty}\left(\left\|D^{\alpha} f\right\|_{(\Phi)} / \sup _{\operatorname{sp}(f)}\left|\xi^{\alpha}\right|\right)^{1 /|\alpha|}<1 .
\end{aligned}
$$

Тем самым мы получили противоречие. Неравенство (29) доказано. 
Наконец, для завершения доказательства теоремы нам осталось показать

$$
(P) \varlimsup_{|\alpha| \rightarrow \infty}\left(\left\|D^{\alpha} f\right\|_{(\Phi)} / \sup _{\operatorname{sp}(f)}\left|\xi^{\alpha}\right|\right)^{1 /|\alpha|} \leqslant 1 .
$$

Докажем (36) методом от противного, допустим, что существуют подпоследовательность $I \subset P$, число $h>1$ и вектор $\beta \geqslant 0,|\beta|=1$, такие, что

$$
\begin{aligned}
& \text { (I) } \lim _{|\alpha| \rightarrow \infty}\left(\left\|D^{\alpha} f\right\|_{(\Phi)} / \sup _{\operatorname{sp}(f)}\left|\xi^{\alpha}\right|\right)^{1 /|\alpha|}>h, \\
& \text { (I) } \lim _{|\alpha| \rightarrow \infty} \frac{\alpha}{|\alpha|}=\beta .
\end{aligned}
$$

Используя обозначения, введенные вьше, имеем такие $1 \leqslant k \leqslant n$ и $i_{1}, \ldots, i_{k} \in$ $\{1, \ldots, n\}$, что $I_{i_{1} \ldots i_{k}}=I \cap T_{i_{1} \ldots i_{k}}$ не ограничено.

Теперь нужно убрать "плохие" точки из $\operatorname{sp}(f)$. Положим

$$
\begin{aligned}
Q= & \left\{\eta \in \mathbb{R}^{n}: \exists\left\{{ }_{m} \xi\right\} \subset \operatorname{sp}(f), \quad m \xi_{j} \neq 0,\right. \\
& \left.j \in\left\{i_{1}, \ldots, i_{k}\right\}, \quad m \geqslant 1, \quad \lim _{m \rightarrow \infty} m \xi=\eta\right\}, \\
Q_{\delta}= & \{x+y: x \in Q, \quad|y|<\delta\}, \quad \delta>0,
\end{aligned}
$$

и $H=\mathbb{R}^{n} \backslash Q$. Тогда $Q$ замкнуто и $H$ и $Q_{\delta}$ открыты.

Следовательно, из $\operatorname{sp}(f) \subset Q_{\delta} \cup H\left(=\mathbb{R}^{n}\right)$ получим

$$
\hat{f}(\xi)=\varphi_{\delta}(\xi) \hat{f}(\xi)+\psi(\xi) \hat{f}(\xi), \quad \varphi_{\delta} \in C_{0}^{\infty}\left(Q_{\delta}\right), \quad \psi \in C_{0}^{\infty}(H) .
$$

Рассуждая, как вьше, мы можем доказать, что $D^{\alpha} F^{-1}(\psi \hat{f})(x) \equiv 0$ для всех $\alpha \in I_{i_{1} \ldots i_{k}}$. Отсюда и из (37) следует, что

$$
\left(I_{i_{1} \ldots i_{k}}\right) \lim _{|\alpha| \rightarrow \infty}\left(\left\|D^{\alpha} F^{-1}\left(\varphi_{\delta} \hat{f}\right)\right\|_{(\Phi)} / \sup _{\operatorname{sp}(f)}\left|\xi^{\alpha}\right|\right)^{1 /|\alpha|}>h
$$

для любого $\delta>0$.

С другой стороны, повторяя рассуждения, проведенные в доказательстве равенства (19), получим

$$
\left(I_{i_{1} \ldots i_{k}}\right) \lim _{|\alpha| \rightarrow \infty} \sup _{Q_{\delta}}\left|\xi^{\alpha}\right|^{1 /|\alpha|}=\sup _{Q_{\delta}}\left|\xi^{\beta}\right| .
$$

Далее, пусть ${ }_{m} \theta \in Q_{1 / m}$, т.е. ${ }_{m} \theta^{\beta}\left|=\sup _{Q_{1 / m}}\right| \xi^{\beta} \mid, m \geqslant 1$. Тогда существуют подпоследовательность $\left\{m_{k}\right\}$ (для простоты обозначения будем считать, что $\left.m_{k}=k, k \geqslant 1\right)$ и точка $\theta^{*} \in Q$ такие, что $m \theta \rightarrow \theta^{*}, m \rightarrow \infty$. Тогда

$$
0<\sup _{Q}\left|\xi^{\beta}\right| \leqslant \lim _{m \rightarrow \infty}\left|{ }_{m} \theta^{\beta}\right|=\left|\theta^{* \beta}\right| .
$$


Рассуждениями, аналогичными приведенным в доказательстве (20), с учетом $\theta^{*} \in Q$ и (38) получим

$$
\left|\theta^{* \beta}\right| \leqslant\left(I_{i_{1} \ldots i_{k}}\right) \underset{|\alpha| \rightarrow \infty}{\lim _{Q}} \sup _{Q}\left|\xi^{\alpha}\right|^{1 /|\alpha|} .
$$

Далее, замечая, что неравенство (27) доказано для произвольной функции Юнга, мы имеем

$$
\left(I_{i_{1} \ldots i_{k}}\right) \varlimsup_{|\alpha| \rightarrow \infty}\left(\left\|D^{\alpha} F^{-1}\left(\varphi_{1 / m} \hat{f}\right)\right\|_{(\Phi)} / \sup _{Q_{1 / m}}\left|\xi^{\alpha}\right|\right)^{1 /|\alpha|} \leqslant 1
$$

для любого $m \geqslant 1$.

Зафиксируем теперь номер $m \geqslant 1$ такой, что $\left|{ }_{m} \theta^{\beta}\right| \leqslant h\left|\theta^{* \beta}\right|$. Тогда, комбинируя (39)-(42), получим

$$
\begin{aligned}
1 & \geqslant\left(I_{i_{1} \ldots i_{k}}\right) \varlimsup_{|\alpha| \rightarrow \infty}\left(\left\|D^{\alpha} F^{-1}\left(\varphi_{1 / m} \hat{f}\right)\right\|_{(\Phi)} / \sup _{Q_{1 / m}}\left|\xi^{\alpha}\right|\right)^{1 /|\alpha|} \\
& =\left(I_{i_{1} \ldots i_{k}}\right) \varlimsup_{|\alpha| \rightarrow \infty}\left\|D^{\alpha} F^{-1}\left(\varphi_{1 / m} \hat{f}\right)\right\|_{(\Phi)}^{1 /|\alpha|} /\left|m \theta^{\beta}\right| \\
& \geqslant\left(I_{i_{1} \ldots i_{k}}\right) \varlimsup_{|\alpha| \rightarrow \infty} h^{-1}\left\|D^{\alpha} F^{-1}\left(\varphi_{1 / m} \hat{f}\right)\right\|_{(\Phi)}^{1 /|\alpha|} /\left|\theta^{* \beta}\right| \\
& \geqslant\left(I_{i_{1} \ldots i_{k}}\right) \varlimsup_{|\alpha| \rightarrow \infty} h^{-1}\left(\left\|D^{\alpha} F^{-1}\left(\varphi_{1 / m} \hat{f}\right)\right\|_{(\Phi)} / \sup _{Q}\left|\xi^{\alpha}\right|\right)^{1 /|\alpha|} \\
& =\left(I_{i_{1} \ldots i_{k}}\right) \lim _{|\alpha| \rightarrow \infty} h^{-1}\left(\left\|D^{\alpha} F^{-1}\left(\varphi_{1 / m} \hat{f}\right)\right\|_{(\Phi)} / \sup _{\operatorname{sp}(f)}\left|\xi^{\alpha}\right|\right)^{1 /|\alpha|}>1,
\end{aligned}
$$

что невозможно. Теорема 2 полностью доказана.

ЗАмечание 5. Равенство (10) показывает, что, зная "дальние" от начала координат точки спектра, мы можем охарактеризовать поведение последовательности $\left\|D^{\alpha} f\right\|_{(\Phi)}, \quad \alpha \geqslant 0$, не вычисляя никаких производных. Следует отметить, что здесь никакого ограничения на геометрию спектра не предполагается. Тонкость полученных результатов заключается в том, что здесь мы изучим поведение последовательности норм $\left\|D^{\alpha} f\right\|_{(\Phi)},|\alpha| \geqslant 0$, в зависимости от носителя преобразования Фурье самой функций $f(x)$, которьй имеет, вообще говоря, произвольную геометрию.

ЗАмечАние 6 . Теорема 2 верна и для случаев дробных производных. Равенство (10) неверно в случае, когда $\operatorname{sp}(f)$ не ограничен. В то же время справедлива

Tеорема 3. Пусть $\Phi(t)$ - произвольная функиия Юнга, $f(x) \in L_{\Phi}\left(\mathbb{R}^{n}\right)$ u $\operatorname{sp}(f)$ ограничен по переменным $\xi_{1}, \ldots, \xi_{k} \quad(1 \leqslant k \leqslant n)$. Тогда $D^{\nu} f(x) \in$ $L_{\Phi}\left(\mathbb{R}^{n}\right)$ для всех $\nu=\left(\nu_{1}, \ldots, \nu_{k}, 0, \ldots, 0\right) \in \mathbb{Z}_{+}^{n} u$

$$
\lim _{|\nu| \rightarrow \infty}\left(\left\|D^{\nu} f\right\|_{(\Phi)} / \sup _{\operatorname{sp}(f)}\left|\xi^{\nu}\right|\right)^{1 /|\nu|}=1 .
$$

Для одномерного случая имеется более сильный результат (см. [15]), метод доказательства которого сушественно отличается от [16]. 
ТЕОРема 4. Пусть $0=n_{0}<n_{1}<\cdots$ - некоторая последовательность чельх чисел, $\Phi(t)$ - произвольная функция Юнга и $D^{n_{k}} f(x) \in L_{\Phi}(\mathbb{R}), \quad k=$ $0,1, \ldots$ Тогда всегда существует предел

$$
d_{f}=\lim _{k \rightarrow \infty}\left\|D^{n_{k}} f\right\|_{(\Phi)}^{1 / n_{k}}
$$

причем $d_{f}=\sigma_{f}=\sup \{|\xi|: \xi \in \operatorname{sp}(f)\}$.

Tеорема 5. Пусть $K$ - произвольное компактное множество в $\mathbb{R}^{n} u$ $\Phi(t)$ - произвольная функция Юнга. Тогда для любого $\epsilon>0$ существует такая постоянная $C_{\epsilon}$, что

$$
\left\|D^{\alpha} f\right\|_{(\Phi)} \leqslant C_{\epsilon}(1+\epsilon)^{|\alpha|}\left(\sup _{K}\left|\xi^{\alpha}\right|\right)\|f\|_{(\Phi)}
$$

для всех $\alpha \geqslant 0$ u $f \in \mathfrak{M}_{K \Phi}$.

ДоказАТЕЛЬСтво. Образуем семейство линейных непрерывных в $\mathfrak{M}_{K \Phi}$ операторов

$$
T_{\alpha}(f)=D^{\alpha} f(x) /(1+\epsilon)^{|\alpha|} \sup _{K}\left|\xi^{\alpha}\right|, \quad \alpha \geqslant 0 .
$$

Тогда в силу теоремы 2 множество $\left\{T_{\alpha}(f): \alpha \geqslant 0\right\}$ ограничено в $\mathfrak{M}_{K \Phi}$ для любой функции $f \in \mathfrak{M}_{K \Phi}$. Следовательно, оно равностепенно непрерьвно в силу теоремы Банаха-Штейнгауза. Теорема 5 доказана.

Рассмотрим теперь соответствуюшие результаты для периодических функций.

Пусть $\mathbb{T}^{n}-n$-мерньй тор. Обозначим через $L_{\Phi}\left(\mathbb{T}^{n}\right)$ пространство периодических функций периода $2 \pi$ по каждой переменной таких, что

$$
\||u|\|_{(\Phi)}=\inf \left\{\lambda>0: \int_{\mathbb{T}^{n}} \Phi(|u(x)| / \lambda) d x \leqslant 1\right\}<\infty .
$$

Методом разложения в ряд Фурье можем доказать следующие результаты.

Tеорема 6. Пусть $g \in L_{\Phi}\left(\mathbb{T}^{n}\right), g(x) \not \equiv 0 u \operatorname{sp}(g)$ ограничен. Тогда

$$
\lim _{|\alpha| \rightarrow \infty}\left(\left\|\left|D^{\alpha} g\right|\right\|_{(\Phi)} / \sup _{k \in \operatorname{sp}(g)}\left|k^{\alpha}\right|\right)^{1 /|\alpha|}=1,
$$

әде $k \in \mathbb{Z}^{n}$.

ТЕОРема 7. Пусть I - некоторое неограниченное семейство мультииндексов, $\Phi(t)$ - произвольная функиия Юнга, $f \in L_{\Phi}\left(\mathbb{T}^{n}\right)$ - такая функиия, что ее обобщенные производные $D^{\alpha} f(x)$ принадлежсат $L_{\Phi}\left(\mathbb{T}^{n}\right)$ для всех $\alpha \in I$. Тогда

$$
\lim _{|\alpha| \rightarrow \infty}\left(\left\|\left|D^{\alpha} f\right|\right\|_{(\Phi)} /\left|k^{\alpha}\right|\right)^{1 /|\alpha|} \geqslant 1
$$

для произвольной точки $k \in \mathrm{sp}(f)$. Это неравенство точно. 


\section{§ 3. Неравенство Никольского в норме Люксембурга}

Важный цикл работ С. М. Никольского и его школы по изучению классов функций многих переменных связан, в частности, с неравенством Никольского для тригонометрических полиномов и целых функций экспоненциального типа. Это неравенство дает возможность разработать методы теории приближения функций многих переменных, с помошью которых и устанавливается связь между дифференциальными свойствами функции в одной лебеговой метрике с подобными свойствами этой функции в другой лебеговой метрике.

Напомним теперь неравенство Никольского. Пусть $1 \leqslant p \leqslant q \leqslant \infty$. Тогда

$$
\left\|t_{m}\right\|_{q, 2 \pi} \leqslant 2^{n}\left(\prod_{j=1}^{n} m_{j}\right)^{1 / p-1 / q}\left\|t_{m}\right\|_{p, 2 \pi}
$$

для тригонометрических полиномов вида

$$
t_{m}(x)=\sum_{j_{1}=-m_{1}}^{m_{1}} \ldots \sum_{j_{n}=-m_{n}}^{m_{n}} c_{\left(j_{1}, \ldots, j_{n}\right)} \exp \left(i\left(j_{1} x_{1}+\cdots+j_{n} x_{n}\right)\right)
$$

и

$$
\|f\|_{q} \leqslant 2^{n}\left(\prod_{j=1}^{n} \nu_{j}\right)^{1 / p-1 / q}\|f\|_{p}
$$

для целых функций экспоненциального типа $\nu$.

Неравенства Никольского привлекали внимание многих математиков таких, как А. Зигмунд [17], И. И. Ибрагимов [3]-[5], Р. Нессель и Дж. Вилмс [18], [19], Г. Трибель [20], [21], В.И. Буренков [6] и др.

Неравенства Никольского симметричных пространств рассматривались в рабо$\operatorname{Tax}[22]-[24]$.

В этом параграфе мы попытаемся установить неравенство Никольского для норм Орлича. Сложность этой задачи связана хотя бы с тем, что нам трудно сравнивать (в явном виде) функции Юнга. Отметим, что пространства Орлича, по нашему определению, не всегда являются симметричными.

ОПРЕДЕЛЕнИЕ 1. Функция $A(t):[0,+\infty) \rightarrow[0,+\infty]$ назьвается квазивыликлой, если

$$
A(\lambda t) \leqslant \lambda A(t), \quad 0 \leqslant \lambda \leqslant 1, \quad t \geqslant 0 .
$$

Ясно, что все функции Юнга являются квазивыпуклыми.

Квазивьпуклая функция $A(t)$ называется тривиальной, если $A(t)=+\infty$ для всех $t>0$. Очевидно, что $\lim _{t \rightarrow 0} A(t)=0$, если $A(t)$ - нетривиальная квазивыпуклая функция.

ОПРЕДЕЛЕНИЕ 2. Пусть $\Phi(t), \Psi(t)$ - функции Юнга. Будем говорить, что $\Psi(t)$ мажорирует $\Phi(t)$, если существует нетривиальная квазивыпуклая функция $A(t)$ такая, что

$$
\Phi(t) \leqslant \Psi(A(t)), \quad t \geqslant 0 .
$$


ПРИмеР 1. Пусть $1 \leqslant p \leqslant q<\infty$ и $\Phi(t)=t^{q}, \Psi(t)=t^{p}, t \geqslant 0$. Тогда

$$
\Phi(t)=\left(t^{q / p}\right)^{p}=\Psi\left(t^{q / p}\right), \quad t \geqslant 0 .
$$

Следовательно, $\Psi(t)$ мажорирует $\Phi(t)$ (здесь $A(t)=t^{q / p}$ ).

Пусть $C_{K \Phi}$ - точная константа в неравенстве

$$
\|f\|_{\infty} \leqslant C_{K \Phi}\|f\|_{(\Phi)} \quad \forall f \in \mathfrak{M}_{K \Phi} .
$$

Справедлива следующая теорема.

Teоpema 8. Пусть $\Psi(t)$ мажсорирует $\Phi(t)$. Тогда

$$
\|f\|_{(\Phi)} \leqslant \frac{A\left(C_{K \Phi}\right)}{C_{K \Phi}}\|f\|_{(\Psi)}
$$

для всех $f \in \mathfrak{M}_{K \Psi}$, где $A(t)$ удовлетворяет (44).

ДоказАтельство. Пусть $f \in \mathfrak{M}_{K \Psi}$. Докажем сначала, что

для некоторого числа $M>0$.

$$
\int \Psi\left(A\left(\frac{|f(x)|}{M}\right)\right) d x \leqslant 1
$$

Действительно, выберем достаточно большое число $M>0$ такое, что

$$
C_{K \Psi}^{-1} A\left(C_{K \Psi}\left(\|f\|_{(\Psi)}+1\right) / M\right) \leqslant 1 .
$$

Тогда из (43) и $|f(x)| \leqslant C_{K \Psi}\|f\|_{(\Psi)}$ следует, что

$$
\begin{aligned}
\Psi\left(A\left(\frac{|f(x)|}{M}\right)\right) & =\Psi\left(A\left(\frac{|f(x)|}{C_{K \Psi}\left(\|f\|_{(\Psi)}+1\right)} \frac{C_{K \Psi}\left(\|f\|_{(\Psi)}+1\right)}{M}\right)\right) \\
& \leqslant \Psi\left(\frac{|f(x)|}{C_{K \Psi}\left(\|f\|_{(\Psi)}+1\right)} A\left(\frac{C_{K \Psi}\left(\|f\|_{(\Psi)}+1\right)}{M}\right)\right) \\
& \leqslant \Psi\left(\frac{|f(x)|}{\|f\|_{(\Psi)}+1}\right) .
\end{aligned}
$$

Отсюда получим $f \in \mathfrak{M}_{K \Phi}$ и (46), так как

$$
\int \Psi\left(\frac{|f(x)|}{\|f\|_{(\Psi)}+1}\right) d x \leqslant 1
$$

Зафиксируем произвольное число $\lambda>0$ такое, что

$$
\int \Psi\left(A\left(\frac{|f(x)|}{\lambda}\right)\right) d x \leqslant 1
$$

Тогда $\lambda \geqslant\|f\|_{(\Phi)}$. Отсюда, учитывая

$$
|f(x)| \leqslant C_{K \Phi}\|f\|_{(\Phi)} \leqslant \lambda C_{K \Phi},
$$

получим

$$
\Psi\left(A\left(\frac{|f(x)|}{\lambda}\right)\right)=\Psi\left(A\left(\frac{|f(x)|}{\lambda C_{K \Phi}} C_{K \Phi}\right)\right) \leqslant \Psi\left(\frac{|f(x)|}{\lambda C_{K \Phi}} A\left(C_{K \Phi}\right)\right) .
$$

Следовательно,

$$
\begin{aligned}
\|f\|_{(\Phi)} & \leqslant \inf \left\{\lambda>0: \int \Psi\left(A\left(\frac{|f(x)|}{\lambda}\right)\right) d x \leqslant 1\right\} \\
& \leqslant \inf \left\{\lambda>0: \int \Psi\left(\frac{|f(x)|}{\lambda C_{K \Phi}} A\left(C_{K \Phi}\right)\right) d x \leqslant 1\right\}=\frac{A\left(C_{K \Phi}\right)}{C_{K \Phi}}\|f\|_{(\Psi)} .
\end{aligned}
$$

Теорема доказана. 
ЗАМЕЧАНИЕ 7. Если мы знаем только, что

$$
\|f\|_{\infty} \leqslant C\|f\|_{(\Phi)} \quad \forall f \in \mathfrak{M}_{K \Phi}
$$

то из доказательства теоремы 8 следует

$$
\|f\|_{(\Phi)} \leqslant \frac{A(C)}{C}\|f\|_{(\Psi)}
$$

для всех $f \in \mathfrak{M}_{K \Psi}$. Тогда из свойства (43) следует

$$
\frac{A\left(C_{K \Phi}\right)}{C_{K \Phi}} \leqslant \frac{A(C)}{C} .
$$

ЗАмечание 8. Рассмотрим случай $\Phi(t)=t^{q}, \Psi(t)=t^{p}, 1 \leqslant p<q<\infty$. Тогда по результатам С. М. Никольского [1], [2] и И.И. Ибрагимова [3]-[5] имеем для всех $f \in \mathfrak{M}_{\nu p}$

$$
\begin{aligned}
& \|f\|_{\infty} \leqslant 2^{n}\left(\nu_{1} \ldots \nu_{n}\right)^{1 / q}\|f\|_{q} \\
& \|f\|_{\infty} \leqslant\left(\left(\frac{s}{\pi}\right)^{n} \nu_{1} \ldots \nu_{n}\right)^{1 / q}\|f\|_{q}
\end{aligned}
$$

где $s$ - наименьшее целое число, большее либо равное $q / 2$. Отсюда из (47) получим

$$
\begin{aligned}
& \|f\|_{q} \leqslant\left(2^{n}\left(\nu_{1} \ldots \nu_{n}\right)^{1 / q}\right)^{q / p-1}\|f\|_{p} \\
& \|f\|_{q} \leqslant\left(\left(\left(\frac{s}{\pi}\right)^{n} \nu_{1} \ldots \nu_{n}\right)^{1 / q}\right)^{q / p-1}\|f\|_{p} .
\end{aligned}
$$

Оказывается, что константа, стоящая в неравенстве (48), будет меньшей, чем соответствующая константа в неравенстве Никольского, если $q<2 p$, а константа, стоящая в неравенстве (49), совпадает с константой Ибрагимова [3]-[5].

ОПРЕДЕЛЕНИЕ 3 . Пусть $\Phi(t), \Psi(t)$ - функции Юнга и $C>0$. Говорим, что $\Psi(t)$ $C$-мажсорирует $\Phi(t)$, если существуют нетривиальная квазивьпуклая функция $A(t)$ и число $C^{*}>C$ такие, что

$$
\Phi(t) \leqslant \Psi(A(t)), \quad 0 \leqslant t<C^{*}
$$

Имеем следующий более сильный результат.

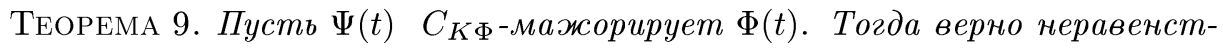
во (45). 
ДокАЗАТЕЛЬСТво. Пусть $f \in \mathfrak{M}_{K \Psi}$. Тогда, повторяя первую часть доказательства теоремы 8 , получим $f \in \mathfrak{M}_{K \Phi}$.

Далее, выберем число $\epsilon_{0}>0$ такое, что

$$
C^{*}\left(\|f\|_{(\Phi)}-\epsilon_{0}\right)=C_{K \Phi}\|f\|_{(\Phi)} .
$$

Тогда из определения $C_{K \Phi}$ следует, что

$$
|f(x)|<C^{*}\left(\|f\|_{(\Phi)}-\epsilon\right), \quad x \in \mathbb{R}^{n},
$$

для всех $0<\epsilon<\epsilon_{0}$. Отсюда получим

$$
\Phi\left(\frac{|f(x)|}{\|f\|_{(\Phi)}-\epsilon}\right) \leqslant \Psi\left(A\left(\frac{|f(x)|}{\|f\|_{(\Phi)}-\epsilon}\right)\right), \quad x \in \mathbb{R}^{n}
$$

для любого $0<\epsilon<\epsilon_{0}$. Отсюда, учитывая

$$
\int \Phi\left(\frac{|f(x)|}{\|f\|_{(\Phi)}-\epsilon}\right) d x>1
$$

что вытекает из определения $\|f\|_{(\Phi)}$, получим

$$
\int \Psi\left(A\left(\frac{|f(x)|}{\|f\|_{(\Phi)}-\epsilon}\right)\right) d x>1
$$

для любого $0<\epsilon<\epsilon_{0}$. Следовательно,

$$
\|f\|_{(\Phi)} \leqslant \inf \left\{\lambda>0: \int \Psi\left(A\left(\frac{|f(x)|}{\lambda}\right)\right) d x \leqslant 1\right\} .
$$

Дальнейшие рассуждения проводятся, как в доказательстве теоремы 8. Теорема доказана.

Аналогично можем доказать следующий более точный результат.

Tеорема 10. Пусть $\Psi(t) \quad C_{f}$-мажсорирует $\Phi(t), \quad f \in L_{\Psi}\left(\mathbb{R}^{n}\right)$ u $\operatorname{sp}(f)$ ограничен. Тогда

$$
\|f\|_{(\Phi)} \leqslant \frac{A\left(C_{f}\right)}{C_{f}}\|f\|_{(\Psi)}
$$

əде $C_{f}=\|f\|_{\infty} /\|f\|_{(\Phi)}$.

Для получения дальнейших неравенств Никольского мы введем понятие порядка квазивыпуклой функции $A(t)$.

ОПРЕДЕЛЕНИЕ 4 . Пусть $C>0$. Назовем супремум всех чисел $p \geqslant 0$ таких, что

$$
A(\lambda C) \leqslant \lambda^{p} A(C)
$$

для всех $0 \leqslant \lambda \leqslant 1, C$-порядком квазивыпуклой функции $A(t)$ и обозначим его через $\operatorname{ord} A$.

Из квазивыпуклости $A(t)$ следует, что ord $A \geqslant 1$. Ясно, что $A(\lambda C) \leqslant \lambda^{\operatorname{ord}} A A(C)$ для всех $0 \leqslant \lambda \leqslant 1$. 


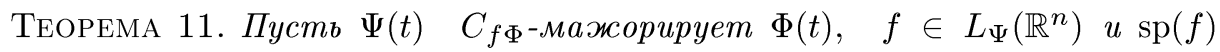
ограничен. Тогда

$$
\|f\|_{(\Phi)} \leqslant \frac{A^{1 / \operatorname{ord} A}\left(C_{f \Phi}\right)}{C_{f \Phi}} C_{f \Psi_{1}}^{\mathrm{ord} A-1}\|f\|_{(\Psi)}
$$

где $C_{f \Phi}=\|f\|_{\infty} /\|f\|_{(\Phi)}, \quad C_{f \Psi_{1}}=\|f\|_{\infty} /\|f\|_{\left(\Psi_{1}\right)}, \quad \Psi_{1}(t)=\Psi\left(t^{\operatorname{ord} A}\right) u \operatorname{ord} A$

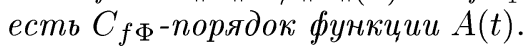

ДокАЗАТЕЛЬСТво. Как мыпоказалив доказательстве теоремы $8, f \in L_{\Phi}\left(\mathbb{R}^{n}\right)$. С другой стороны, из предположения теоремы следует, что

$$
\Phi(t) \leqslant \Psi(A(t)), \quad 0 \leqslant t<C^{*},
$$

где $C_{f \Phi}<C^{*}$ - некоторое число.

Далее, выберем число $\epsilon_{0}>0$ такое, что

$$
C^{*}\left(\|f\|_{(\Phi)}-\epsilon_{0}\right)=C_{f \Phi}\|f\|_{(\Phi)} .
$$

Тогда из определения числа $C_{f \Phi}$ вытекает

$$
|f(x)|<C^{*}\left(\|f\|_{(\Phi)}-\epsilon\right), \quad x \in \mathbb{R}^{n},
$$

для всех $0<\epsilon<\epsilon_{0}$. Отсюда и из (51) получим

$$
\Phi\left(\frac{|f(x)|}{\|f\|_{(\Phi)}-\epsilon}\right) \leqslant \Psi\left(A\left(\frac{|f(x)|}{\|f\|_{(\Phi)}-\epsilon}\right)\right), \quad x \in \mathbb{R}^{n},
$$

для любого $0<\epsilon<\epsilon_{0}$. Следовательно, учитьвая

$$
\int \Phi\left(\frac{|f(x)|}{\|f\|_{(\Phi)}-\epsilon}\right) d x>1 \quad \forall \epsilon>0
$$

получим

$$
\int \Psi\left(A\left(\frac{|f(x)|}{\|f\|_{(\Phi)}-\epsilon}\right)\right) d x>1
$$

для всякого $0<\epsilon<\epsilon_{0}$. Отсюда получим

$$
\|f\|_{(\Phi)} \leqslant \inf \left\{\lambda>0: \int \Psi\left(A\left(\frac{|f(x)|}{\lambda}\right)\right) d x \leqslant 1\right\} .
$$

Зафиксируем теперь произвольное число $\lambda>0$ такое, что

$$
\int \Psi\left(A\left(\frac{|f(x)|}{\lambda}\right)\right) d x \leqslant 1
$$

Тогда из (52) вытекает $\lambda \geqslant\|f\|_{(\Phi)}$. Следовательно,

$$
A\left(\frac{|f(x)|}{\lambda}\right)=A\left(\frac{|f(x)|}{\lambda C_{f \Phi}} C_{f \Phi}\right) \leqslant\left(\frac{|f(x)|}{\lambda C_{f \Phi}}\right)^{\operatorname{ord} A} A\left(C_{f \Phi}\right) .
$$


Отсюда, учитывая (52), получим

$$
\begin{aligned}
\|f\|_{(\Phi)} & \leqslant \inf \left\{\lambda>0: \int \Psi\left(A\left(\frac{|f(x)|}{\lambda}\right)\right) d x \leqslant 1\right\} \\
& \leqslant \inf \left\{\lambda>0: \int \Psi\left(\left(\frac{|f(x)|}{\lambda C_{f \Phi}}\right)^{\operatorname{ord} A} A\left(C_{f \Phi}\right)\right) d x \leqslant 1\right\} \\
& =\inf \left\{\lambda>0: \int \Psi\left(\left(\frac{|f(x)| A^{1 / \operatorname{ord} A}\left(C_{f \Phi}\right)}{\lambda C_{f \Phi}}\right)^{\operatorname{ord} A}\right) d x \leqslant 1\right\} \\
& =\frac{A^{1 / \operatorname{ord} A}\left(C_{f \Phi}\right)}{C_{f \Phi}} \inf \left\{\lambda>0: \int \Psi_{1}\left(\frac{|f(x)|}{\lambda}\right) d x \leqslant 1\right\},
\end{aligned}
$$

ибо $\Psi_{1}(t)=\Psi\left(t^{\text {ord } A}\right)$ является функцией Юнга и $\|\beta f\|_{\left(\Psi_{1}\right)}=|\beta|\|f\|_{\left(\Psi_{1}\right)}$ для любого числа $\beta$.

Тем самым мы доказали, что

$$
\|f\|_{(\Phi)} \leqslant \frac{A^{1 / \operatorname{ord} A}\left(C_{f \Phi}\right)}{C_{f \Phi}}\|f\|_{\left(\Psi_{1}\right)} .
$$

Отсюда, применяя теорему 10 для пары функций $\Psi_{1}(t)$ и $\Psi(t)$, получим

$$
\|f\|_{(\Phi)} \leqslant \frac{A^{1 / \operatorname{ord} A}\left(C_{f \Phi}\right)}{C_{f \Phi}} C_{f \Psi_{1}}^{\mathrm{ord} A-1}\|f\|_{(\Psi)} .
$$

Теорема доказана.

Рассмотрим случай $\Phi(t)=t^{q}, \Psi(t)=t^{p}, 1 \leqslant p<q<\infty$. Тогда $A(t)=t^{q / p}$ и $C$-порядок функции $A(t)$ равняется $q / p$ для всех $C>0$. В этом случае константа в неравенстве (50) равна $C_{f q}^{q / p}$, ибо тогда $\Psi_{1}(t) \equiv \Phi(t)$, где $C_{f q}=\|f\|_{\infty} /\|f\|_{q}$.

Следует отметить, что в отличие от известных неравенств Никольского, константы, фигурируюшие в теоремах 10, 11, зависят от самой функции (а не от класca).

Рассмотрим теперь неравенство Никольского для тригонометрических полиноMOB.

Пусть $K$ - некоторое компактное подмножество в $\mathbb{R}^{n}$. Обозначим через $\mathscr{P}_{K \Phi}$ пространство всех функций $f \in L_{\Phi}\left(\mathbb{T}^{n}\right)$ таких, что $\operatorname{sp}(f) \subset K$. Тогда легко доказать, что справедливо непрерьвное вложение $\mathscr{P}_{K \Phi} \subset \mathscr{P}_{K \infty}$, где $\mathscr{P}_{K \infty}$ есть пространство всех ограниченных периодических функций со спектром на $K$.

Рассуждениями, аналогичными приведенным выше, можем доказать следуюшие результаты.

TeOPEMA 12. Пусть $\Psi(t) C_{K \Phi}^{*}$-мажорирует $\Phi(t)$. Тогда

$$
\|\| f\left\|_{(\Phi)} \leqslant \frac{A\left(C_{K \Phi}^{*}\right)}{C_{K \Phi}^{*}} \mid\right\| f \|_{(\Psi)}
$$

для всех $f \in \mathscr{P}_{K \Psi}$, где $C_{K \Phi}^{*}-$ точная константа неравенства

$$
|\|f\||_{\infty} \leqslant C|\|f\||_{(\Phi)} .
$$


Teорема 13. Пусть $\Psi(t) \quad C_{f \Phi}^{*}$-мажсорирует $\Phi(t), \quad f \in L_{\Psi}\left(\mathbb{T}^{n}\right) u \operatorname{sp}(f)$ ограничен. Тогда

$$
\|\| f\left\|_{(\Phi)} \leqslant \frac{A\left(C_{f \Phi}^{*}\right)}{C_{f \Phi}^{*}} \mid\right\| f \|_{(\Psi)},
$$

əде $C_{f \Phi}^{*}=\left|\|f\|_{\infty} /\right|\|f\|_{(\Phi)}$.

Teоpema 14. Пусть $\Psi(t) \quad C_{f \Phi}^{*}$-мажсорирует $\Phi(t), \quad f \in L_{\Psi}\left(\mathbb{T}^{n}\right) u \operatorname{sp}(f)$ ограничен. Тогда

$$
\|\| f\left\|_{(\Phi)} \leqslant \frac{A^{1 / \operatorname{ord} A}\left(C_{f \Phi}^{*}\right)}{C_{f \Phi}^{*}} C_{f \Psi_{1}}^{\text {ord } A-1} \mid\right\| f \|_{(\Psi)},
$$

əде $C_{f \Phi}^{*}=\left|\|f\|_{\infty} /\|f\|\left\|_{(\Phi)}, \quad C_{f \Psi_{1}}^{*}=|\|f\||_{\infty} /\right\|\|f\|_{\left(\Psi_{1}\right)}, \quad \Psi_{1}(t)=\Psi_{1}\left(t^{\text {ord } A}\right) u\right.$ ord $A$ есть $C_{f \Phi}^{*}$-порядок функции $A(t)$.

\section{§4. Некоторые применения}

Пусть $0 \leqslant \lambda_{\alpha} \leqslant \infty, \alpha \in \mathbb{Z}_{+}^{n}$ и $G\left\{\lambda_{\alpha}\right\}=\bigcap_{\alpha \geqslant 0}\left\{\xi \in \mathbb{R}^{n}:\left|\xi^{\alpha}\right| \leqslant \lambda_{\alpha}\right\}$.

ОПРЕДЕЛЕНИЕ 5. Назовем $G\left\{\lambda_{\alpha}\right\}$ множеством, порождаемым числовой последовательностью $\left\{\lambda_{\alpha}\right\}$.

Ясно, что $G\left\{\lambda_{\alpha}\right\}$ замкнуто, $\left(r_{1} \xi_{1}, \ldots, r_{n} \xi_{n}\right) \in G\left\{\lambda_{\alpha}\right\}$, если $\xi \in G\left\{\lambda_{\alpha}\right\}$ и $\left|r_{j}\right| \leqslant 1$, $j=1, \ldots, n$, и

$$
G\left\{\lambda_{\alpha}\right\}=G\left\{\sup _{\xi \in G\left\{\lambda_{\alpha}\right\}}\left|\xi^{\alpha}\right|\right\} .
$$

Множество $G\left\{\lambda_{\alpha}\right\}$ компактно, если, например, $\lambda_{\alpha}<\infty \quad \forall \alpha \geqslant 0$.

Заметим, что $G\left\{\lambda_{\alpha}\right\}$ может быть невыпуклым. Например, пусть $n=2$ и

$$
\lambda_{(i, j)}=2^{|i-j|} \quad \forall i, j \in \mathbb{Z}_{+} .
$$

Невыпуклым является множество, называемое крестом гиперболы,

$$
G\left\{\lambda_{(i, j)}\right\}=\left\{(x, y) \in \mathbb{R}^{2}:|x y| \leqslant 1, \quad|x| \leqslant 2, \quad|y| \leqslant 2\right\} .
$$

Пусть $K \subset \mathbb{R}^{n}$. Если положить $g(K)=G\left\{\sup _{K}\left|\xi^{\alpha}\right|\right\}$, то $K \subset g(K)$. Назовем $g(K) g$-оболочкой множества $K$.

ОПРЕДЕЛЕНИЕ 6 . Будем говорить, что множество $K$ обладает $g$-свойством, если $K=g(K)$.

Ясно, что любое множество, порождаемое числовой последовательностью $G\left\{\lambda_{\alpha}\right\}$, обладает $g$-свойством и, очевидно, наоборот.

ЛЕмма 5. Пусть $I$ - некоторое семейство индексов и $K_{i}=g\left(K_{i}\right), \quad i \in I$. Тогда $\bigcap_{i \in I} K_{i}$ также обладает g-свойством. 
ДоКАЗАТЕЛЬСтво. Пусть $x \in g\left(\bigcap_{i \in I} K_{i}\right)$ и $j \in I$. Тогда для любого $\alpha \in \mathbb{Z}_{+}^{n}$ имеем

$$
\left|x^{\alpha}\right| \leqslant \sup \left\{\left|\xi^{\alpha}\right|: \xi \in \bigcap_{i \in I} K_{i}\right\} \leqslant \sup \left\{\left|\xi^{\alpha}\right|: \xi \in K_{j}\right\}
$$

Следовательно, $x \in g\left(K_{j}\right)=K_{j}$. Поэтому $x \in \bigcap_{i \in I} K_{j}$.

Возникает вопрос: каждое ли компактное множество $K$ со свойством

$$
x \in K, \quad-1 \leqslant \lambda_{j} \leqslant 1, \quad j=1, \ldots, n \Longrightarrow\left(\lambda_{1} x_{1}, \ldots, \lambda_{n} x_{n}\right) \in K
$$

обладает $g$-свойством? Оказывается, что ответ отрицателен. Действительно, пусть $K$ - такое произвольное подмножество креста параболы $G=\left\{(x, y) \in \mathbb{R}^{2}\right.$ : $|x y| \leqslant 1, \quad|x| \leqslant 2, \quad|y| \leqslant 2\}$, что $K$ обладает свойством $(53), K \neq G$, и $K$ содержит в себе точки $\left(\frac{1}{2}, 2\right)$ и $\left(2, \frac{1}{2}\right)$. Такие множества $K$, очевидно, сушествуют, например $K=\left\{|x| \leqslant 2, \quad|y| \leqslant \frac{1}{2}\right\} \cup\left\{|x| \leqslant \frac{1}{2},|y| \leqslant 2\right\}$. Тогда

$$
\sup _{K}\left|x^{i} y^{j}\right|=2^{|i-j|}=\sup _{G}\left|x^{i} y^{j}\right|
$$

для всех целых чисел $i, j \geqslant 0$. Следовательно, $K$ не обладает $g$-свойством.

Лемма 6. Каждое симметричное выпуклое компактное множество обладает g-свойством.

ДоКАЗАТЕЛЬСТвО. Пусть $K$ - некоторый симметричный выпуклый компакт в $\mathbb{R}^{n}$. Тогда легко видеть, что $K$ удовлетворяет (53). Далее, пусть $y \notin K$. Тогда сушествует вектор $a \in \mathbb{R}^{n}$ такой, что

$$
a y>\sup _{x \in K} a x,
$$

что следует из выпуклости $K$, где $a \xi=a_{1} \xi_{1}+\cdots+a_{n} \xi_{n}$. Из (53) имеем

$$
\sup _{x \in K} a x=\sup _{x \in K}\left(\left|a_{1} x_{1}\right|+\cdots+\left|a_{n} x_{n}\right|\right) .
$$

И также из (53) следует, что $y \notin K$ тогда и только тогда, когда $\left(\left|y_{1}\right|, \ldots,\left|y_{n}\right|\right) \notin K$. Следовательно, нам достаточно рассмотреть только случай $y \geqslant 0$. Тогда в силу $(54),(55)$ можем выбрать такой вектор $a$, что $a_{j} \geqslant 0$ и $a_{j}=0$, если $y_{j}=0$, $j=1, \ldots, n$.

Считаем теперь для удобства, что $y_{j}>0, j=1, \ldots, n$.

Пусть $x \in K_{+}=\{x \in K: x \geqslant 0\}$. Тогда из одного известного классического неравенства следует

$$
\begin{aligned}
\left(\frac{a x}{a y}\right)^{a y} & =\left(\frac{a_{1} y_{1} x_{1} / y_{1}+\cdots+a_{n} y_{n} x_{n} / y_{n}}{a_{1} y_{1}+\cdots+a_{n} y_{n}}\right)^{a_{1} y_{1}+\cdots+a_{n} y_{n}} \\
& \geqslant\left(\frac{x_{1}}{y_{1}}\right)^{a_{1} y_{1}} \cdots\left(\frac{x_{n}}{y_{n}}\right)^{a_{n} y_{n}}
\end{aligned}
$$

Следовательно, учитывая (54), получим

$$
1>\sup _{x \in K_{+}}\left(\frac{x_{1}}{y_{1}}\right)^{a_{1} y_{1}} \ldots\left(\frac{x_{n}}{y_{n}}\right)^{a_{n} y_{n}} .
$$


Аппроксимируя $a_{j} y_{j}$ рациональными числами, имеем

$$
1>\sup _{x \in K_{+}}\left(\frac{x_{1}}{y_{1}}\right)^{p_{1} / q_{1}} \cdots\left(\frac{x_{n}}{y_{n}}\right)^{p_{n} / q_{n}},
$$

где $p_{j} \geqslant 0, q_{j}>0, j=1, \ldots, n,-$ некоторые целые числа. Следовательно, из (53) имеем

$$
y^{\alpha}>\sup _{x \in K_{+}} x^{\alpha}=\sup _{x \in K}\left|x^{\alpha}\right|
$$

где $\alpha_{j}=q_{1} \ldots q_{n} p_{j} / q_{j}, j=1, \ldots, n$. Это означает, что $y \notin g(K)$ для любого $y \notin K$. Доказательство закончено.

Докажем теперь невыпуклый случай теоремы Пэли-Винера-Шварца, характеризующий связь между поведением последовательности норм производных функции и носителем ее преобразования Фурье.

Для множества, порождаемого числовой последовательностью $G\left\{\lambda_{\alpha}\right\}$, ясно, что если существуют $m \geqslant 1$ и $\beta \geqslant 0$ такие, что $\lambda_{\beta}^{m}<\lambda_{m \beta}$, то множество $G\left\{\lambda_{\alpha}\right\}$ не изменяется при замене $\lambda_{m \beta} \rightarrow \lambda_{\beta}^{m}$. Следовательно, для определения $G\left\{\lambda_{\alpha}\right\}$ всегда можно предполагать, что

$$
\lambda_{\alpha}^{m} \geqslant \lambda_{m \alpha} \quad \forall m \geqslant 1, \quad \alpha \geqslant 0 .
$$

ОПРЕДЕЛЕНИЕ 7. Будем говорить, что последовательность $\left\{\lambda_{\alpha}\right\}$ nравильна, если $\left\{\lambda_{\alpha}\right\}$ удовлетворяет условию (56).

Теорема 15. Пусть $\Phi(t)$ - произвольная функиия Юнга, $f \in L_{\Phi}\left(\mathbb{R}^{n}\right)$, $G\left\{\lambda_{\alpha}\right\}$ ограничено и последовательность $\left\{\lambda_{\alpha}\right\}$ правильна. Тогда для того, чтобы $\operatorname{sp}(f) \subset G\left\{\lambda_{\alpha}\right\}$, необходимо и достаточно, чтобы выполнялось следующее условие:

$$
\varlimsup_{|\alpha| \rightarrow \infty}\left(\left\|D^{\alpha} f\right\|_{(\Phi)} / \lambda_{\alpha}\right)^{1 /|\alpha|} \leqslant 1
$$

ДокАЗАТЕЛЬСтво. Пусть $\operatorname{sp}(f) \subset G\left\{\lambda_{\alpha}\right\}$. Тогда имеем

$$
\sup _{\operatorname{sp}(f)}\left|\xi^{\alpha}\right| \leqslant \lambda_{\alpha}, \quad \alpha \geqslant 0
$$

Следовательно, из теоремы 2 получим (57).

Обратно, пусть (57) выполнено. Заметим, что неравенство (11) справедливо и для случая, когда $\mathrm{sp}(f)$ не ограничен. Следовательно, из $(11),(57)$ и правильности последовательности $\left\{\lambda_{\alpha}\right\}$ легко вытекает, что $\operatorname{sp}(f)$ ограничен. Отсюда, учитывая теоремы 2 , получим

$$
\varlimsup_{|\alpha| \rightarrow \infty}\left(\sup _{\operatorname{sp}(f)}\left|\xi^{\alpha}\right| / \lambda_{\alpha}\right)^{1 /|\alpha|} \leqslant 1 .
$$

Следовательно, для любого $\epsilon>0$ сушествует номер $N<\infty$ такой, что

$$
\sup _{\operatorname{sp}(f)}\left|\xi^{\alpha}\right| \leqslant(1+\epsilon)^{|\alpha|} \lambda_{\alpha}, \quad|\alpha| \geqslant N .
$$


С другой стороны, последовательность $\left\{\lambda_{\alpha}\right\}$ правильна. Следовательно,

$$
\sup _{\operatorname{sp}(f)}\left|\xi^{\alpha}\right| \leqslant(1+\epsilon)^{|\alpha|} \lambda_{\alpha}
$$

для всех $\alpha \geqslant 0$. Отсюда получаем

$$
\operatorname{sp}(f) \subset(1+\epsilon) G\left\{\lambda_{\alpha}\right\}
$$

Устремляя $\epsilon$ к 0 , получим $\mathrm{sp}(f) \subset G\left\{\lambda_{\alpha}\right\}$. Теорема доказана.

Применим теперь полученные результаты для изучения некоторых вопросов теории пространств Соболева-Орлича бесконечного порядка, возникающих при изучении нелинейных дифференциальных уравнений бесконечного порядка с коэффициентами произвольного роста. Теория пространства бесконечного порядка была введена Ю.А. Дубинским и изучена им, а также Г. С. Балашовой, Чан Дык Ваном, Л. И. Клениной, Ю. А. Коняевым, А. Я. Кобиловым, С. Р. Умаровым, А.Н. Агаджановым, Л.Н. Грошевым, автором и другими, кто рассматривал следуюшие вопросы: нетривиальность, теория следов, связь с краевыми задачами, теоремы вложения, геометрические свойства и т.п. (см., например, [25]-[27] и библиографию в них). Следует отметить, что теория функциональных пространств бесконечного порядка отличается от теории пространств конечного порядка хотя бы тем, что совершенно нетривиален вопрос о сушествовании ненулевого элемента функциональных пространств бесконечного порядка (т.е. вопрос о нетривиальности). Положительный ответ на него играет решающую роль в теории краевых задач для дифференциальных уравнений бесконечного порядка. Задача о нахождении решений краевых задач для уравнений бесконечного порядка содержательна, если соответствующие энергетические пространства нетривиальны (см., например, [28]-[31]).

Пусть $I$ - некоторьй неограниченный набор целочисленных индексов $\alpha=$ $\left(\alpha_{1}, \ldots, \alpha_{n}\right), \alpha_{j} \geqslant 0, j=1, \ldots, n, \Phi_{\alpha}(t), \alpha \in I,-$ произвольные функции Юнга. Тогда

$$
W^{\infty} L\left\{\Phi_{\alpha}, \mathbb{R}^{n}\right\}=\left\{f(x) \in S^{\prime}: \sum_{\alpha \in I}\left\|D^{\alpha} f\right\|_{\left(\Phi_{\alpha}\right)}<\infty\right\}
$$

называется пространством Соболева-Орлича бесконечного порядка.

Найдем условие нетривиальности $W^{\infty} L\left\{\Phi_{\alpha}, \mathbb{R}^{n}\right\}$. Предположим, что $0 \in I$ и $\Phi_{0}(t)>0, t>0$, ибо в противном случае $W^{\infty} L\left\{\Phi_{\alpha}, \mathbb{R}^{n}\right\}$ нетривиально. Применяя теорему 2, можно доказать следующую теорему [31].

Теорема 16. Для того чтобы $W^{\infty} L\left\{\Phi_{\alpha}, \mathbb{R}^{n}\right\}$ было нетривиальным. необходимо и достаточно, чтобы существовали такие числа $C, q>0$, что

$$
\sum_{\alpha \in I} \Phi_{\alpha}\left(C q^{|\alpha|}\right)<\infty
$$

Опишем теперь свойства функций из $W^{\infty} L\left\{\Phi_{\alpha}, \mathbb{R}^{n}\right\}$. 
ОПРЕДЕЛЕНИЕ 8 . Пусть выполнено условие (58). Обозначим через $C_{\Phi}$ объединение всех точек $\xi \in \mathbb{R}^{n}$ таких, что

$$
\sum_{\alpha \in I} \Phi_{\alpha}\left(C_{\xi} \sup _{x \in G_{\xi}}\left|x^{\alpha}\right|\right)<\infty
$$

для некоторых области $G_{\xi} \ni \xi$ и числа $C_{\xi}>0$. Ясно, что $G_{\Phi}$ открыто, непусто и симметрично относительно всех осей координат.

Теорема 17. Пусть выполнено условие (58). Тогда

$$
F^{-1}\left[C_{0}^{\infty}\left(G_{\Phi}\right)\right] \subset W^{\infty} L\left\{\Phi_{\alpha}, \mathbb{R}^{n}\right\}
$$

u $W^{\infty} L\left\{\Phi_{\alpha}, \mathbb{R}^{n}\right\}$ не содержит ни одной функиии $F^{-1} g(x)$, если $g \in C_{0}^{\infty}\left(\mathbb{R}^{n}\right)$ $u \operatorname{supp} g \cap\left(\mathbb{R}^{n} \backslash \bar{G}_{\Phi}\right) \neq \varnothing$.

ДокАЗАТЕЛЬСТВО. Пусть $\varphi(\xi) \in C_{0}^{\infty}\left(G_{\Phi}\right)$. Тогда для любой точки $\xi \in \operatorname{supp} \varphi$ сушествует ограниченная область $G_{\xi} \ni \xi$, удовлетворяющая условию (59). Отсюда в силу компактности $\operatorname{supp} \varphi$ сушествуют номер $M<\infty$, число $C>0$ и ограниченные области $G_{j}$ такие, что

$$
\operatorname{supp} \varphi \subset \bigcup_{j=1}^{M} G_{j}
$$

и

$$
\sum_{\alpha \in I} \Phi_{\alpha}\left(C \sup _{G_{j}}\left|\xi^{\alpha}\right|\right)<\infty, \quad j=1, \ldots, M
$$

Далее, для каждой области $G$ определим

$$
G^{*}=\left\{\left(t_{1} \xi_{1}, \ldots, t_{n} \xi_{n}\right): \xi \in G, \quad-1 \leqslant t_{j} \leqslant 1, \quad j=1, \ldots, n\right\} .
$$

Тогда $G^{*}$ также является областью и для всех $\alpha \geqslant 0$

$$
\sup _{G}\left|\xi^{\alpha}\right|=\sup _{G^{*}}\left|\xi^{\alpha}\right| .
$$

В силу компактности $\operatorname{supp} \varphi$ имеем для некоторого числа $0<\lambda<1$

$$
\operatorname{supp} \varphi \subset \lambda \bigcup_{j=1}^{M} G_{j}^{*} .
$$

Пусть $\psi(x)=\left(F^{-1} \varphi\right)(x)$. Тогда $\psi \in \mathfrak{M}_{K 1}$, где для кратности обозначим $K=\operatorname{supp} \varphi$. Будем считать для удобства, что $C=1$.

Докажем теперь, что $\psi \in W^{\infty} L\left\{\Phi_{\alpha}, \mathbb{R}^{n}\right\}$. Действительно, для любых $\alpha, \beta \geqslant 0$

$$
\begin{aligned}
\left|x^{\beta} D^{\alpha} \psi(x)\right| & \leqslant \int_{K}\left|D^{\beta}\left(\xi^{\alpha} \varphi(\xi)\right)\right| d \xi \\
& \leqslant \sum_{\gamma \leqslant \beta, \alpha} \frac{\beta !}{\gamma !(\beta-\alpha) !} \prod_{k=1}^{n} \alpha_{k} \ldots\left(\alpha_{k}-\gamma_{k}+1\right) \int_{K}\left|\xi^{\alpha-\gamma} D^{\beta-\gamma} \varphi(\xi)\right| d \xi .
\end{aligned}
$$


Отсюда, учитьвая

$$
\prod_{k=1}^{n} \alpha_{k} \ldots\left(\alpha_{k}-\gamma_{k}+1\right) \leqslant|\alpha|^{|\gamma|}, \quad \sum_{\gamma \leqslant \beta} \frac{\beta !}{\gamma !(\beta-\gamma) !}=2^{|\beta|},
$$

получим для всех $x \in \mathbb{R}^{n}, \alpha \geqslant 0$ и $|\beta| \leqslant 2 n$

$$
\left|x^{\beta} D^{\alpha} \psi(x)\right| \leqslant 2^{2 n}|\alpha|^{2 n} C_{2 n} \max \left\{\sup _{K}\left|\xi^{\alpha-\gamma}\right|: \gamma \leqslant \alpha, \quad|\gamma| \leqslant 2 n\right\},
$$

где

$$
C_{2 n}=\max \left\{\int_{K}\left|D^{\beta-\gamma} \varphi(\xi)\right| d \xi: \gamma \leqslant \beta, \quad|\beta| \leqslant 2 n\right\} .
$$

С другой стороны, из (25) имеем

$$
\lim _{|\alpha| \rightarrow \infty}\left(\max \left\{\sup _{K}\left|\xi^{\alpha-\gamma}\right|: \gamma \leqslant \alpha, \quad|\gamma| \leqslant 2 n\right\}\right)^{1 /|\alpha|} / \sup _{K}\left|\xi^{\alpha}\right|^{1 /|\alpha|}=1 .
$$

Следовательно, для любого $\epsilon>0$ существует число $C_{\epsilon}$ такое, что

$$
\left(1+x_{1}^{2}\right) \ldots\left(1+x_{n}^{2}\right)\left|D^{\alpha} \psi(x)\right| \leqslant 2^{2 n} C_{2 n} C_{\epsilon}|\alpha|^{2 n}(1+\epsilon)^{|\alpha|} \sup _{K}\left|\xi^{\alpha}\right|
$$

для всех $x \in \mathbb{R}^{n}, \alpha \geqslant 0$.

Из (62) и (63) получаем для $G^{*}=\bigcup_{j=1}^{M} G_{j}^{*}$

$$
\left(1+x_{1}^{2}\right) \ldots\left(1+x_{n}^{2}\right)\left|D^{\alpha} \psi(x)\right| \leqslant 2^{2 n} C_{2 n} C_{\epsilon}|\alpha|^{2 n}(1+\epsilon)^{|\alpha|} \lambda^{|\alpha|} \sup _{G^{*}}\left|\xi^{\alpha}\right| .
$$

Выберем теперь число $\epsilon>0$ такое, что $(1+\epsilon) \lambda<1$, и пусть $(1+\epsilon) \lambda=\lambda_{1} q$, где $0<\lambda_{1}, q<1$. Тогда из

$$
\lim _{|\alpha| \rightarrow \infty}|\alpha|^{2 n}(1+\epsilon)^{|\alpha|} \lambda_{1}^{|\alpha|}=0
$$

следует, что

$$
\left|D^{\alpha} \psi(x)\right| \leqslant\left(1+x_{1}^{2}\right)^{-1} \ldots\left(1+x_{n}^{2}\right)^{-1} q^{|\alpha|} \sup _{G^{*}}\left|\xi^{\alpha}\right|
$$

для всех $|\alpha| \geqslant N_{1}$ и $x \in \mathbb{R}^{n}$. Отсюда получим для $|\alpha| \geqslant N_{1}$

$$
\begin{aligned}
& \left\|D^{\alpha} \psi\right\|_{\left(\Phi_{\alpha}\right)}=\inf \left\{\gamma>0: \int_{\mathbb{R}^{n}} \Phi_{\alpha}\left(\frac{\left|D^{\alpha} \psi(x)\right|}{\gamma}\right) d x \leqslant 1\right\} \\
& \quad \leqslant \inf \left\{\gamma>0: \int_{\mathbb{R}^{n}} \Phi_{\alpha}\left(q^{|\alpha|} \sup _{G^{*}}\left|\xi^{\alpha}\right| / \gamma\left(1+x_{1}^{2}\right) \ldots\left(1+x_{n}^{2}\right)\right) d x \leqslant 1\right\} \\
& \quad \leqslant \inf \left\{\gamma>0: \Phi_{\alpha}\left(q^{|\alpha|} \sup _{G^{*}}\left|\xi^{\alpha}\right| / \gamma\right) \int_{\mathbb{R}^{n}}\left(1+x_{1}^{2}\right)^{-1} \ldots\left(1+x_{n}^{2}\right)^{-1} d x \leqslant 1\right\} \\
& \quad=\inf \left\{\gamma>0: \pi^{n} \Phi_{\alpha}\left(q^{|\alpha|} \sup _{G^{*}}\left|\xi^{\alpha}\right| / \gamma\right) \leqslant 1\right\} .
\end{aligned}
$$


С другой стороны, из (60) и (61) имеем

$$
\sum_{\alpha \in I} \Phi_{\alpha}\left(\sup _{G^{*}}\left|\xi^{\alpha}\right|\right) \leqslant \sum_{\alpha \in I} \max _{1 \leqslant j \leqslant M} \Phi_{\alpha}\left(\sup _{G_{j}}\left|\xi^{\alpha}\right|\right) \leqslant \sum_{j=1}^{M} \sum_{\alpha \in I} \Phi_{\alpha}\left(\sup _{G_{j}}\left|\xi^{\alpha}\right|\right)<\infty .
$$

Следовательно, существует номер $N_{2}<\infty$ такой, что

$$
\Phi_{\alpha}\left(\sup _{G^{*}}\left|\xi^{\alpha}\right|\right) \leqslant \pi^{-n}, \quad|\alpha| \geqslant N_{2}
$$

Значит,

$$
\pi^{n} \Phi_{\alpha}\left(q^{|\alpha|} \sup _{G^{*}}\left|\xi^{\alpha}\right| / q^{|\alpha|}\right) \leqslant 1, \quad|\alpha| \geqslant N_{2} .
$$

Последние неравенства и (64) означают, что

$$
\left\|D^{\alpha} \psi\right\|_{\left(\Phi_{\alpha}\right)} \leqslant q^{|\alpha|}
$$

для всех $|\alpha| \geqslant N_{0}=\max \left\{N_{1}, N_{2}\right\}$.

С другой стороны, ясно из (63), что $D^{\alpha} \psi(x) \in L_{\Phi}\left(\mathbb{R}^{n}\right)$ для любой функции Юнга $\Phi(t)$ и для всех $\alpha \geqslant 0$. Следовательно, учитывая (65), получим $\psi(x) \in$ $W^{\infty} L\left\{\Phi_{\alpha}, \mathbb{R}^{n}\right\}$. Первая часть теоремы 17 доказана.

Докажем теперь вторую часть. Пусть $g(\xi) \in C_{0}^{\infty}\left(\mathbb{R}^{n}\right)$ есть такая функция, что $\operatorname{supp} g \cap\left(\mathbb{R}^{n} \backslash \bar{G}_{\Phi}\right)=\varnothing$. Тогда из открытости $\mathbb{R}^{n} \backslash \bar{G}_{\Phi}$ следует, что сушествуют точка $\xi \in \operatorname{supp} g, \xi_{j} \neq 0, j=1, \ldots, n$, и окрестность $U_{\xi} \subset \mathbb{R}^{n} \backslash \bar{G}_{\Phi}, \xi \in U_{\xi}$.

С другой стороны, из $\xi \notin \bar{G}_{\Phi}$ следует, что существует число $r<1$ такое, что для любого $C<\infty$ и для любой области $G_{\xi} \ni \xi$

$$
\sum_{\alpha \in I} \Phi_{\alpha}\left(C r^{|\alpha|} \sup _{G_{\xi}}\left|x^{\alpha}\right|\right)=\infty
$$

ибо в противном случае имеем $\forall r<1 \exists C_{r}, \exists G_{\xi} \ni \xi$ :

$$
\sum_{\alpha \in I} \Phi_{\alpha}\left(C_{r} r^{|\alpha|} \sup _{G_{\xi}}\left|x^{\alpha}\right|\right)<\infty
$$

T.e.

$$
\sum_{\alpha \in I} \Phi_{\alpha}\left(C_{r} \sup _{r G_{\xi}}\left|x^{|\alpha|}\right|\right)<\infty .
$$

Заметим, что $\xi \in r G_{\xi}$ для некоторого $r<1$. Зафикиируем такое число $r$ и положим $Q=r G_{\xi}$. Тогда имеем

$$
\sum_{\alpha \in I} \Phi_{\alpha}\left(C_{r} \sup _{Q}\left|x^{\alpha}\right|\right)<\infty
$$

значит, $\xi \in G_{\Phi}$, что невозможно. Равенство (66) доказано.

7

Серия математическая, №2 
Докажем теперь $f=F^{-1} g \notin W^{\infty} L\left\{\Phi_{\alpha}, \mathbb{R}^{n}\right\}$ методом от противного. Пусть $f \in W^{\infty} L\left\{\Phi_{\alpha}, \mathbb{R}^{n}\right\}$. Не теряя обшности, считаем, что $\sum_{\alpha \in I}\left\|D^{\alpha} f\right\|_{\left(\Phi_{\alpha}\right)} \leqslant 1$. Положим

$$
f_{h}(x)=\frac{1}{\operatorname{mes} B(0, h)} \int_{B(0, h)} f(x+t) d t, \quad h>0 .
$$

Тогда из неравенства Йенсена следует, что

$$
\Phi_{\alpha}\left(\left|D^{\alpha} f_{h}(x)\right|\right) \leqslant \frac{1}{\operatorname{mes} B(0, h)} \int_{B(0, h)} \Phi_{\alpha}\left(\left|D^{\alpha} f(x+t)\right|\right) d t .
$$

С другой стороны, можем считать, что все функции $\Phi_{\alpha}(t), \alpha \in I$, непрерывны слева. Действительно, если $\Phi(t)$ не является непрерьвной слева, то сушествует точка $t_{0}>0$ такая, что

$$
\lim _{t \rightarrow t_{0^{-}}} \Phi(t)<\Phi\left(t_{0}\right) \leqslant \infty, \quad \Phi(t)=\infty \text { для } t>t_{0} .
$$

Положим

$$
\Psi(t)= \begin{cases}\Phi(t), & t \neq t_{0}, \\ \lim _{t \rightarrow t_{0^{-}}} \Phi(t), & t=t_{0} .\end{cases}
$$

Тогда $\Psi(t)$ является непрерывной слева функцией Юнга и

$$
\|\cdot\|_{(\Psi)}=\|\cdot\|_{(\Phi)} .
$$

Следовательно, можем заменить $\Phi(t)$ на $\Psi(t)$.

Тогда из

$$
\int \Phi_{\alpha}\left(\left|D^{\alpha} f(x)\right|\right) d x \leqslant\left\|D^{\alpha} f\right\|_{\left(\Phi_{\alpha}\right)},
$$

так как $\left\|D^{\alpha} f\right\|_{\left(\Phi_{\alpha}\right)} \leqslant 1$ (см. [7]-[9]), следует

$$
\sum_{\alpha \in I} \Phi_{\alpha}\left(\left\|D^{\alpha} f_{h}\right\|_{\infty}\right) \leqslant \frac{1}{\operatorname{mes} B(0, h)}
$$

Из определения $f_{h}(x)$ ясно, что $f_{h} \rightarrow f$ слабо в $S^{\prime}$ при $h \rightarrow 0$. Следовательно, $\hat{g}_{h} \rightarrow \hat{f}$ слабо в $S^{\prime}$. Отсюда следует, что точка $\xi$, определенная выше, принадлежит к supp $\hat{f}_{h}$ для достаточно малого $h>0$, ибо $\xi \in \operatorname{supp} \hat{f}=\operatorname{supp} g$.

Зафиксируем такое достаточно малое число $h>0$, что $\xi \in \operatorname{supp} \hat{f}_{h}$. Тогда из (11) следует

$$
\lim _{|\alpha| \rightarrow \infty}\left(\left\|D^{\alpha} f_{h}\right\|_{\infty} /\left|\xi^{\alpha}\right|\right)^{1 /|\alpha|} \geqslant 1 .
$$

Следовательно, для любого $\epsilon>0$ сушествует число $C_{\epsilon}>0$ такое, что

$$
\left\|D^{\alpha} f_{h}\right\|_{\infty} \geqslant C_{\epsilon}(1-\epsilon)^{|\alpha|}\left|\xi^{\alpha}\right|, \quad \alpha \in I .
$$

С другой стороны, из $\xi_{j} \neq 0, j=1, \ldots, n$, вытекает, что для любого $\lambda>1$ сушествует такая окрестность $G$ точки $\xi$, что

$$
\sup _{x \in G}\left|x^{\alpha}\right| \leqslant \lambda^{|\alpha|}\left|\xi^{\alpha}\right|
$$


для всех $\alpha \geqslant 0$.

Выберем теперь $\lambda>1$ и $\epsilon>0$ такие, что $\lambda^{-1}(1-\epsilon) \geqslant r$. Тогда из (67)-(69) вытекает

$$
\begin{aligned}
& \sum_{\alpha \in I} \Phi_{\alpha}\left(C_{\epsilon} r^{|\alpha|} \sup _{G}\left|\xi^{\alpha}\right|\right) \leqslant \sum_{\alpha \in I} \Phi_{\alpha}\left(C_{\epsilon} \lambda^{-|\alpha|}(1-\epsilon)^{|\alpha|} \sup _{G}\left|\xi^{\alpha}\right|\right) \\
& \leqslant \sum_{\alpha \in I} \Phi_{\alpha}\left(C_{\epsilon}(1-\epsilon)^{|\alpha|}\left|\xi^{\alpha}\right|\right) \leqslant \sum_{\alpha \in I} \Phi_{\alpha}\left(\left\|D^{\alpha} f_{h}\right\|_{\infty}\right) \leqslant \frac{1}{\operatorname{mes} B(0, h)}
\end{aligned}
$$

что невозможно в силу (66). Теорема доказана.

ТЕОРема 18. Пусть выполнено условие (58). Пространство $W^{\infty} L\left\{\Phi_{\alpha}, \mathbb{R}^{n}\right\}$ содержит все функиии $f \in L_{1}\left(\mathbb{R}^{n}\right) c \operatorname{sp}(f) \subset G_{\Phi}$, но не содержит ни одной функиии $g \in L_{1}\left(\mathbb{R}^{n}\right)$ c $\operatorname{sp}(g) \cap\left(\mathbb{R}^{n} \backslash \bar{C}_{\Phi}\right) \neq \varnothing$. Более того, если $\Phi_{\alpha}(t)>0, t>0$, при некотором $\alpha \in I$, то $\operatorname{sp}(g) \cap\left(\mathbb{R}^{n} \backslash \bar{C}_{\Phi}\right)=\varnothing$ для любой $g \in W^{\infty} L\left\{\Phi_{\alpha}, \mathbb{R}^{n}\right\}$. Последний факт неверен в противном случае.

ДоказАтельство. Пусть $f \in L_{1}\left(\mathbb{R}^{n}\right)$ и $\operatorname{sp}(f) \subset G_{\Phi}$. Выберем функцию $\widehat{\varphi}(\xi) \in$ $C_{0}^{\infty}\left(G_{\Phi}\right)$ такую, что $\widehat{\varphi}(\xi)$ равна единище в некоторой окрестности $\operatorname{sp}(f)$. Тогда из неравенств

$$
\left\|D^{\alpha} f\right\|_{\left(\Phi_{\alpha}\right)}=\left\|f * D^{\alpha} \varphi\right\|_{\left(\Phi_{\alpha}\right)} \leqslant 2\|f\|_{1}\left\|D^{\alpha} \varphi\right\|_{\left(\Phi_{\alpha}\right)}, \quad \alpha \in I
$$

и теоремы 17 немедленно получим $f \in W^{\infty} L\left\{\Phi_{\alpha}, \mathbb{R}^{n}\right\}$, ибо $\varphi \in W^{\infty} L\left\{\Phi_{\alpha}, \mathbb{R}^{n}\right\}$.

То, что $W^{\infty} L\left\{\Phi_{\alpha}, \mathbb{R}^{n}\right\}$ не содержит ни одной функции $g \in L_{1}\left(\mathbb{R}^{n}\right)$ с $\operatorname{sp}(g) \cap$ $\left(\mathbb{R}^{n} \backslash \bar{C}_{\Phi}\right) \neq \varnothing$, доказывается рассуждениями, совершенно аналогичными проведенными в доказательстве второй части теоремы 17 , с учетом того, что на основании следствия 1 каждая точка $\xi \in \mathrm{sp}(g)$ обладает последовательностью точек $\operatorname{sp}(g)$ с ненулевыми компонентами, сходящихся к $\xi$.

Далее, пусть $\Phi_{\beta}(t)>0, t>0$, при некотором $\beta \in I$ и $g(x) \in W^{\infty} L\left\{\Phi_{\alpha}, \mathbb{R}^{n}\right\}$. Нам достаточно рассмотреть только случай $g(x) \not \equiv 0$. Тогда из $\left\|D^{\beta} g\right\|_{\left(\Phi_{\beta}\right)}<\infty$, $\|g\|_{\left(\Phi_{0}\right)}<\infty$ и теоремы 1 следует, что:

если $D^{\beta} g(x) \equiv 0$, то $\operatorname{sp}(g) \subset\{0\} ;$ отсюда $\operatorname{sp}(g) \cap\left(\mathbb{R}^{n} \backslash \bar{C}_{\Phi}\right)=\varnothing ;$

если $D^{\beta} g(x) \not \equiv 0$, то ограничение $\hat{g}(\xi)$ на любой окрестности любой точки $\operatorname{sp}(g)$ не сосредоточено на гиперплоскостях $\xi_{j}=0, j=1, \ldots, n$.

Следовательно, можем доказать наше утверждение рассуждениями, аналогичными проведенными в доказательстве второй части теоремы 17.

Для завершения построим один контрпример. Пусть $n \geqslant 2$,

$$
I_{j}=\left\{(\underbrace{m, \ldots, m}_{j-1}, m^{2}, m, \ldots, m): m \geqslant 0\right\}, \quad I=\bigcup_{j=1}^{n} I_{j}
$$

$\Phi_{\alpha}(t)=0$ для $0 \leqslant t \leqslant 1$ и $\Phi_{\alpha}(t)=\infty$ для $t>1$ при всех $\alpha \in I$ (т.е. при $\|\cdot\|_{\left(\Phi_{\alpha}\right)}=$ $\left.\|\cdot\|_{\infty}\right)$. Тогда легко видеть, что $G_{\Phi}=M$, где $M=\left\{\xi \in \mathbb{R}^{n}:\left|\xi_{j}\right|<1, j=\right.$ $1, \ldots, n\}$. Действительно, если $x \notin \bar{M}$, то сушествует индекс $1 \leqslant j \leqslant n$ такой, что 
$\left|x_{j}\right|>1$. Тогда для любой окрестности $U_{x}$ и для любого числа $0<C<\infty$ имеем некоторое $\theta>0$ такое, что для всех $\alpha \in I_{j}$

$$
\sup _{\xi \in U_{x}}\left|\xi^{\alpha}\right| \geqslant \theta^{(n-1) m}\left|x_{j}\right|^{m^{2}}, \quad \alpha=(\underbrace{m, \ldots, m}_{j-1}, m^{2}, m, \ldots, m) .
$$

Отсюда, учитывая $\theta^{n-1}\left|x_{j}\right|^{m} \rightarrow \infty, m \rightarrow \infty$, получим $\Phi_{\alpha}\left(C \sup _{\xi \in U_{x}}\left|\xi^{\alpha}\right|\right)=\infty$ при достаточно большом $|\alpha|, \alpha \in I_{j}$. Это означает, что $x \notin G_{\Phi}$.

Более того, из $\left|x_{j}\right|>1$ следует, что существует такая окрестность $V$ точки $x$, что $\left|y_{j}\right|>1$ для любой точки $y \in V$. Следовательно, $V \cap G_{\Phi}=\varnothing$. Значит, $x \notin \bar{G}_{\Phi}$. Тем самым мы доказали, что

$$
\bar{G}_{\Phi} \subset \bar{M}=\left\{\xi \in \mathbb{R}^{n}:\left|\xi_{j}\right| \leqslant 1, \quad j=1, \ldots, n\right\} .
$$

$\mathrm{C}$ другой стороны, из выбора наших функций $\Phi_{\alpha}(t)$ очевидно, что $M \subset G_{\Phi}$. Следовательно, $G_{\Phi}=M$, ибо множество $G_{\Phi}$ всегда открыто.

Наконец, заметим, что любая функция

$$
\varphi(x)=\varphi\left(x_{1}, \ldots, x_{n-1}\right)=F^{-1} \widehat{\varphi}(\xi), \quad \widehat{\varphi}(\xi) \in C_{0}^{\infty}\left(\mathbb{R}^{n-1}\right),
$$

принадлежит $W^{\infty} L\left\{\Phi_{\alpha}, \mathbb{R}^{n}\right\}$, ибо $D^{\alpha} \varphi(x) \equiv 0$ для всех $\alpha \in I,|\alpha|>0$. Это означает, что свойство $\operatorname{sp}(g) \cap\left(\mathbb{R}^{n} \backslash \bar{C}_{\Phi}\right)=\varnothing$ неверно. Теорема доказана.

ЗАмЕчАниЕ 9. Мы показали, что при $n \geqslant 2$ утверждение $\operatorname{sp}(g) \cap\left(\mathbb{R}^{n} \backslash \bar{C}_{\Phi}\right)=\varnothing$ может быть неверным, если для любого $\alpha \in I$ существует число $t_{\alpha}>0$ такое, что $\Phi_{\alpha}\left(t_{\alpha}\right)=0$. При $n=1$ имеем более сильный результат.

Теорема 19. Пусть выполнено соответствующее условие (58). Тогда имеем $\operatorname{sp}(g) \cap\left(\mathbb{R}^{1} \backslash \bar{C}_{\Phi}\right)=\varnothing$ для любой $g \in W^{\infty} L\left\{\Phi_{m}, \mathbb{R}^{1}\right\}$.

ДокАЗАТЕЛЬСТво. Докажем методом от противного. Пусть сушествуют функция $g(x) \in W^{\infty} L\left\{\Phi_{m}, \mathbb{R}^{1}\right\}$ и точка $\xi \in \operatorname{sp}(g)$ такие, что $\xi \in \mathbb{R}^{1} \backslash \bar{G}_{\Phi}$. Тогда $G_{\Phi}=(-b, b), 0<b<\infty$ и $|\xi|>b$.

Определим

$$
g_{h}(x)=\frac{1}{h} \int_{0}^{h} g(x+t) d t, \quad h>0 .
$$

Тогда, как показано в доказательстве теоремы 17 , имеем

$$
\sum_{m \in I} \Phi_{m}\left(\left\|D^{m} g_{h}\right\|_{\infty}\right) \leqslant \frac{1}{h}<\infty
$$

ибо можем считать, что $\sum_{m \in I}\left\|D^{m} g\right\|_{\left(\Phi_{m}\right)} \leqslant 1$. Далее, из слабой сходимости $\hat{g}_{h} \rightarrow \hat{g}$ в $S^{\prime}$ следует, что $\xi \in \operatorname{supp} \hat{g}_{h}$ при достаточно малом $h>0$. Зафиксируем такое $h>0$, что $\xi \in \operatorname{supp} \hat{g}_{h}$. Тогда из (11) вытекает

$$
\varliminf_{m \rightarrow \infty}\left\|D^{m} g_{h}\right\|_{\infty}^{1 / m} \geqslant|\xi| .
$$

Для определенности считаем, что $\xi>0$. Тогда существуют числа $0<\epsilon, C<\infty$ такие, что $\xi-\epsilon>b$ и

$$
\left\|D^{m} g_{h}\right\|_{\infty} \geqslant C(\xi-\epsilon)^{m}, \quad m \geqslant 0 .
$$

Следовательно,

$$
\sum_{m \in I} \Phi_{m}\left(C(\xi-\epsilon)^{m}\right)<\infty
$$

что невозможно, так как $\xi-\epsilon>b$ и $G_{\Phi}=(-b, b)$. Теорема доказана. 
ТеОРема 20. Пусть выполнено условие (58). Тогда

$$
\bar{G}_{\Phi} \subset \cup\left\{\operatorname{sp}(g): g \in W^{\infty} L\left\{\Phi_{\alpha}, \mathbb{R}^{n}\right\}\right\} .
$$

ДоКАЗАТЕЛЬСТВо. Из теоремы 17 имеем

$$
G_{\Phi} \subset \cup\left\{\operatorname{sp}(g): g \in W^{\infty} L\left\{\Phi_{\alpha}, \mathbb{R}^{n}\right\}\right\} .
$$

Далее, пусть $\xi^{0} \in \bar{G}_{\Phi} \backslash G_{\Phi}$. Тогда существует последовательность точек $\left\{\xi^{k}\right\} \subset G_{\Phi}$ такая, что $\xi^{k} \rightarrow \xi^{0}, k \rightarrow \infty$, и $\xi^{k} \neq \xi^{l}, k \neq l$. Пусть числа $\epsilon_{k}>0, k \geqslant 1$, такие, что $B\left(\xi^{k}, \epsilon_{k}\right) \cap B\left(\xi^{l}, \epsilon_{l}\right)=\varnothing$ и $B\left(\xi^{k}, \epsilon_{k}\right) \subset G_{\Phi}, k \geqslant 1$. Выберем функции $\widehat{\varphi}_{k}(\xi) \in C_{0}^{\infty}\left(B\left(\xi^{k}, \epsilon_{k}\right)\right)$ и числа $\gamma_{k}>0$ такие, что $\widehat{\varphi}_{k}\left(\xi^{k}\right) \neq 0, k \geqslant 1$, и

$$
\sum_{k=1}^{\infty} \gamma_{k}\left\|\left|\varphi_{k}\right|\right\|<\infty
$$

Тогда $\psi(x)=\sum_{k=1}^{\infty} \gamma_{k} \varphi_{k}(x) \in W^{\infty} L\left\{\Phi_{\alpha}, \mathbb{R}^{n}\right\}$ и $\xi^{0} \in \operatorname{sp}(\psi)$. Теорема доказана.

\section{Список литературы}

1. Никольский C. M. Неравенства для целых функций конечной степени и их применение в теории дифференцируемых функций многих переменных // Тр. МИАН СССР. 1951. T. 38 . C. $244-278$.

2. Никольский C. M. Приближение функций многих переменных и теоремы вложения. М.: Наука, 1977.

3. Ибрагимов И. И. Экстремальные свойства целых функций конечной степени. Баку: Элм, 1962.

4. Ибрагимов И. И. Экстремальные задачи в одном классе целых функций конечной степени // Изв. АН СССР. Сер. матем. 1959. Т. 23. С. 243-256.

5. Ибрагимов И. И. Некоторые экстремальные задачи в одном классе целых функций конечной степени // Изучения современных проблем конструктивной теории функций. Баку: Изд-во АН АзерССР, 1965. С. 212-219.

6. Буренков В.И. Теоремы вложения и продолжения для классов дифференцируемых функций многих переменных, заданных во всем пространстве // Итоги науки. Матем. анализ. М.: Изд-во ВИНИТИ АН СССР, 1966. С. 71-155.

7. Красносельский М. А., Рутицкий Я.Б. Выпуклые функции и пространства Орлича. М.: Физматгиз, 1958.

8. Adams R. Sobolev spaces. New York: Academic Press, 1975.

9. Luxemburg $W$. Banach function spaces: Thesis. The Netherlands: Technische Hogeschool te Delft, 1955.

10. O'Neil R. Fractional integration in Orlicz space. I // Trans. Amer. Math. Soc. 1965. V. 115. P. $300-328$.

11. Соболев С. Л. Некоторые применения функционального анализа в математической физике. Новосибирск: Изд-во Сиб. отд. АН СССР, 1962.

12. Хёрмандер Л. Анализ линейных дифференциальных операторов с частными производными. Т. 1. М.: Мир, 1986.

13. Владимиров В. С. Обобщенные функции в математической физике. М.: Наука, 1976.

14. Гельфанд И. М., Шилов Г. Е. Обобщенные функции. Вып. 2. Пространства основных и обобщенных функций. М.: Физматгиз., 1958.

15. Ha Huy Bang, Marimoto $M$. The sequence of Luxemburg norms of derivatives // Tokyo J. of Math. 1994. V. 17. № 1. P. 141-147.

16. Ha Huy Bang. A property of infinitely differentiable functions // Proc. Amer. Math. Soc. 1990. V. 108. № 1. P. 73-76. 
17. Szegö G., Zygmund A. On certain mean values of polynomials // J. Analyse Math. 1953. V. 3. P. 225-244.

18. Nessel R.J., Wilmes G. Nikolskii-type inequalities in connection with regular spectral measures // Acta Math. 1979. V. 33. P. 169-182.

19. Nessel R.J., Wilmes G. Nikolskii-type inequalities for trigonometric polynomials and entire functions of exponential type // J. Austral. Math. Soc. 1978. V. 25. P. 7-18.

20. Triebel $H$. General function spaces. II: Inequalities of Plancherel-Polya-Nikolskii-type, $L_{p}$-space of analytic functions: $0<p \leqslant \infty / /$ J. Approximation Theory. 1977. V. 19 . P. 154-175.

21. Triebel H. Theory of function spaces. Basel, Boston, Stuttgart: Birkhäuser, 1983.

22. Родин B. А. Неравенства Джексона и Никольского для тригонометрических полиномов в симметричном пространстве // Труды 7-й зимней школы. Дрогобыч, 1974. М., 1976. C. 133-139.

23. Овчинников В. И. Интерполяционные теоремы, вытекающие из неравенства Гротендика // Функцион. анализ и его прилож. 1976. Т. 10. № 4. С. 45-54.

24. Берколайко М. З., Овчинников В. И. Неравенства для целых функций экспоненциального типа в симметричных пространствах // Тр. МИАН СССР. 1983. Т. 161. С. 3-17.

25. Дубинский Ю. А. Пространства Соболева бесконечного порядка // УМН. 1991. Т. 43. №6. C. $97-131$.

26. Dubinskij Ju. A. Sobolev spaces of infinite order and differential equations. Dordrecht-Boston-Lankaster-Tokyo: Riedel, 1986.

27. Tran Duc Van, Dinh Nho Hao. Differential operators of infinite order with real arguments and their applications. Singapore: World Scientific, 1994.

28. Дубинский Ю. А. Пространства Соболева бесконечного порядка и поведение задач при неограниченном возрастании порядка уравнений // Матем. сб. 1975. Т. 98 (140). № 2. C. $163-184$.

29. Дубинский Ю. А. Нетривиальность пространств Соболева бесконечного порядка в случае полного эвклидова пространства и тора // Матем. сб. 1976. Т. 100(142). №3. C. $436-446$.

30. Чан Дьк Ван. Нелинейные дифференциальные уравнения и функциональные пространства бесконечного порядка. Минск: Изд-во БГУ, 1983.

31. Ха Зуй Банг. Критерии нетривиальности классов, пространств Соболева-Орлича бесконечного порядка в полном евклидовом пространстве // Сиб. матем. журн. 1990. Т. 31. № 1. C. $208-213$.

Институт математики, г. Ханой, СРВ

Поступило в редакцию

20.VI. 1995 